\title{
The functional evolution of termite gut microbiota
}

Jigyasa Arora $^{*}$, Yukihiro Kinjo ${ }^{1}$, Jan Šobotník ${ }^{2}$, Aleš Buček ${ }^{1}$, Crystal Clitheroe ${ }^{1}$, Petr Stiblik ${ }^{3}$, Yves Roisin ${ }^{4}$, Lucia Žifčáková ${ }^{1}$, Yung Chul Park ${ }^{5}$, Ki Yoon Kim ${ }^{5}$, David Sillam-Dussès ${ }^{6,7}$, Vincent Hervé ${ }^{8}$, Nathan Lo ${ }^{9}$, Gaku Tokuda ${ }^{10}$, Andreas Brune ${ }^{8}$, Thomas Bourguignon ${ }^{1,2 *}$

${ }^{1}$ Okinawa Institute of Science \& Technology Graduate University, 1919-1 Tancha, Onna-son, Okinawa, 904-0495, Japan

${ }^{2}$ Faculty of Tropical AgriSciences, Czech University of Life Sciences, Prague, Czech Republic

${ }^{3}$ Faculty of Forestry and Wood Sciences, Czech University of Life Sciences, Prague, Czech Republic

${ }^{4}$ Evolutionary Biology and Ecology, Université Libre de Bruxelles, Belgium

${ }^{5}$ Division of Forest Science, Kangwon National University, Chuncheon, Republic of Korea

${ }^{6}$ Institut de Recherche pour le Développement - Sorbonne Universités, iEES-Paris, U 242, Bondy, France

${ }^{7}$ Université Paris 13 - Sorbonne Paris Cité, LEEC, EA 4443, Villetaneuse, France

${ }^{8}$ Research Group Insect Gut Microbiology and Symbiosis, Max Planck Institute for Terrestrial Microbiology, Marburg, Germany

${ }^{9}$ School of Life and Environmental Sciences, University of Sydney, Sydney, NSW 2006, Australia

${ }^{10}$ Tropical Biosphere Research Center, Center of Molecular Biosciences, University of the Ryukyus, Nishihara, 903-0213 Okinawa, Japan

*Authors for correspondence: Jigyasa Arora, email: arorajigyasa1992@gmail.com; Thomas Bourguignon, e-mails: thomas.bourgui@gmail.com, thomas.bourguignon@ oist.jp; 


\section{SUMMARY}

Termites primarily feed on lignocellulose or soil in association with specific gut microbes. The functioning of the termite gut microbiota is partly understood in a handful of wood-feeding pest species, but remains largely unknown in other taxa. We intend to feel this gap and provide a global understanding of the functional evolution of termite gut microbiota. We sequenced the gut metagenomes of 145 samples representative of the termite diversity. We show that the prokaryotic fraction of the gut microbiota of all termites possesses similar genes for carbohydrate and nitrogen metabolisms, in proportions varying with termite phylogenetic position and diet. The presence of a conserved set of gut prokaryotic genes implies that key nutritional functions were present in the ancestor of modern termites. Furthermore, the abundance of these genes largely correlated with the host phylogeny. Finally, we found that the adaptation to a diet of soil by some termite lineages was accompanied by a change in the stoichiometry of genes involved in important nutritional functions rather than by the acquisition of new genes and pathways. Our results reveal that the composition and function of termite gut prokaryotic communities have been remarkably conserved since termites first appeared $\sim 150$ million years ago. Therefore, the "world smallest bioreactor" has been operating as a multipartite symbiosis composed of termites, archaea, bacteria, and cellulolytic flagellates since its inception.

\section{Keywords}

Isoptera, endosymbionts, metagenomics, vertical inheritance 


\section{INTRODUCTION}

Termites are one of the few animal lineages feeding on substrates distributed along the wood-soil decomposition gradient (Donovan et al., 2001; Bourguignon et al., 2011). Although termites produce their own endogenous cellulases (Watanabe et al., 1998; Tokuda et al., 2004), their ability to decompose wood or soil organic matter largely depends on symbiosis with mutualistic gut microbes (Watanabe and Tokuda, 2010; Brune and Ohkuma, 2011), including bacteria, archaea and, in the case of lower termites, cellulolytic flagellates. The cellulolytic flagellates of termites are typically found nowhere else other than in termite guts and are efficiently transmitted across host generations (Nalepa, 2017; Michaud et al., 2020). Similarly, many of the prokaryotes present in termite guts are found nowhere else in nature (Bourguignon et al., 2018; Hervé et al., 2020). Their vertical mode of inheritance is supported by the observations that differences among termite gut prokaryotic and protist communities tend to increase as phylogenetic distances among termite hosts increase (Rahman et al., 2015; Tai et al., 2015). In addition, the diet of the termite host, which largely correlates with the termite phylogeny (Bourguignon et al., 2011), also shapes the termite gut microbial communities (Dietrich et al., 2014; Mikaelyan et al., 2015). Whether the termite phylogeny is recapitulated by gut microbial functions, as it is recapitulated by the taxonomic composition of microbial communities, remains unknown.

Investigations of termite gut microbe genomes has revealed that, in addition to the production of enzymes involved in lignocellulose digestion, gut microbes have numerous nutritional functions, including nitrogen fixation and recycling abilities that supplement the nitrogen-poor diet of their host (Lilburn et al., 2001; Yamada et al., 2007; Hongoh et al., 2008; Ohkuma, M. \& Brune, 2011). While metagenomics and metatranscriptomics surveys of termite guts have been carried out for an increasingly large number of termite species (Warnecke et al., 2007; He et al., 2013; Liu et al., 2018; Tokuda et al., 2018; Marynowska et al., 2020), often with the prospect of harvesting cellulolytic enzymes able to convert plant biomass into biofuel (e.g. Tartar et al., 2009; Calusinska et al., 2020), there has been a marked sampling bias towards easy-to-sample wood-feeding termite species, and species with pest status. Far less is known about the function and taxonomy of the gut prokaryotic communities of other termite lineages, such as basal wood-feeding lineages, or lineages with soil-feeding habits (Hervé et al., 2020). Because of this gap in our knowledge, it remains largely unclear how the taxonomy and function of gut microbiome has been evolving 
since termites came to be 150 Million years ago (Bourguignon et al., 2015; Bucek et al., 2019). Similarly, how the acquisition of a diet based on soil has affected the taxonomy and function of gut microbial communities remains an open question. A metagenomics survey based on a comprehensive sampling of termites is required to answer these questions.

In this study, we sequenced whole gut metagenomes of 145 termite samples representatives of the phylogenetic and ecological diversity of termites, including many lineages that have remained undocumented. We also sequenced the gut metagenome of one sample of Cryptocercus, the sister group of termites (Lo et al., 2000). We used the assembled prokaryotic contigs of this dataset to determine (1) when important gut prokaryotic pathways involved in nutritional functions were acquired by termites; (2) to which extent termite phylogeny is predictive of gut prokaryote taxonomic and functional composition; and (3) the taxonomic and functional changes experienced by gut prokaryote communities following the acquisition of a diet of soil.

\section{RESULTS AND DISCUSSION}

\section{The taxonomic composition of termite gut prokaryotes}

We sequenced whole gut metagenomes, including the hindgut containing the bulk of the gut microbiota, of 145 termite species (Table S1, Figure S1). This included species from the nine termite families and species from the eight subfamilies of Termitidae (Lo et al., 2000). Our shotgun sequencing approach generated an average of 72.5 million reads per sample that were assembled into an average of 92,237 scaffolds $>1000 \mathrm{bps}$, constituting $63.3 \%$ of mapped reads. The proportions of prokaryotic reads were on average $18.4 \%$ in lower termites and $20.5 \%$ in higher termites.

We used 40 marker genes (Sunagawa et al., 2013; Wu et al., 2013) to determine the taxonomy and estimate the abundance of each major bacterial lineage present in the 129 termite gut metagenome assemblies including upward of 10,000 contigs longer than 1000 bps. Shorter contigs were removed from the analyses. The bacterial community composition and abundance inferred from marker gene data showed similarities at the phylum level to that inferred from 16S rRNA gene amplicon sequences (Figure S2). However, the abundance distribution estimated by both approaches showed some disagreements for several families (Dietrich et al., 2014; Mikaelyan et 
al., 2015; Bourguignon et al., 2018). Notably, Dysgomonadaceae, Ruminococcaceae, Synergistaceae, and Oscillospiraceae occurred at low abundances among the marker genes but were represented by many 16S rRNA gene sequences in most termite species (Dietrich et al., 2014; Mikaelyan et al., 2015; Bourguignon et al., 2018) (Table S2). These discrepancies are likely the result of variation in 16S rRNA gene copy number (Větrovský and Baldrian, 2013; Edgar, 2018), which are higher in these lineages, or are possibly artifacts generated during 16S rRNA gene amplicon PCR cycles. They might also reflect the incomplete coverage of our metagenomes or, to a certain extent, the differences in the databases used for classification.

In total, we identified 114 family-level bacterial lineages, belonging to 19 phyla and represented in the gut of more than 5\% of termite species (Table S3). Many other bacterial family-level lineages were recorded from the gut of no more than a few termite species, and were possibly transient, and not strictly associated with termite guts. We calculated the Moran I index on the abundance of these 114 family-level bacterial lineages to test whether bacterial abundance is correlated with termite phylogeny. We found a phylogenetic autocorrelation signal for 59 of the 114 bacterial lineages, and this signal remained significant at a 5\% false discovery rate (FDR) correction for 27 bacterial lineages, including some of the most abundant bacterial lineages (Figure 1, Table S4). For example, the wood-fiber-associated Fibrobacteraceae (Mikaelyan et al., 2014; Tokuda et al., 2018) are dominant in the gut of Microcerotermes, Nasutitermitinae, and related termite lineages, and are either undetectable or occur at low abundance in the assemblies of other termite lineages. Another example is the Endomicrobiaceae that comprise flagellate-associated (Stingl et al., 2005; Zheng et al., 2015) and free-living Endomicrobia (Ikeda-Ohtsubo et al., 2016; Mikaelyan et al., 2017), which were abundant in lower termites and almost entirely absent in higher termites.

Our dense taxonomic sampling of diverse termite hosts also allowed us to identify bacterial lineages whose association with termites has remained largely unreported. For example, we found that the Holophagaceae, a bacterial family of Acidobacteriota previously reported from the gut of three humus-feeding termite species (Mikaelyan et al., 2015) and two species of Nasutitermitinae (Dietrich et al., 2014), is widely distributed in Nasutitermitinae, Foraminitermitinae, the Cephalotermes-group, and the Pericapritermes-group (Figure 1). Altogether, our results 
demonstrate that termite phylogeny is remarkably predictive of the gut bacterial community composition, as has been demonstrated for termite gut protists (Tai et al., 2015).

Using the same 40 marker genes and 129 metagenome assemblies used for bacteria, we investigated the diversity of gut-associated Archaea across the termite phylogenetic tree. In total, we identified 16 family-level archaeal lineages, including Methanoculleaceae and Methanocorpusculaceae (order Methanomicrobiales), Methanosarcinaceae (order Methanosarcinales), Methanobacteriaceae (order Methanobacteriales), Methanomethylophilaceae (order Methanomassiliicoccales), and UBA233 (class Bathyarchaeia). All but nine family-level lineages were present in the gut of more than $5 \%$ of termite species. The abundance of Methanosarcinaceae, UBA233, and an unclassified family-level lineage of Bathyarchaeia showed significant autocorrelation signals with the termite phylogenetic tree when no FDR correction was applied (Figure 1, Table S4). Bathyarchaeia occurred in the clade of Termitidae excluding Macrotermitinae, Sphaerotermitinae, and Foraminitermitinae confirming previous reports (Loh et al., 2021), and Methanosarcinaceae was found in Macrotermitinae, Nasutitermitinae, and in Cubitermitinae and related termite lineages (Figure 1). Archaea represented in average less than $1 \%$ of the gut prokaryotes in wood-feeding termite species, while their proportion reached $4.6 \%$ in Macrotermitinae and $10.6 \%$ in soil-feeding termite species, and was exceptionally high in the soil-feeding Mimeutermes in which 59.8\% of the marker genes were assigned to Bathyarchaeia. Our results are in line with the higher archaeal-to-bacterial ratios reported in soil-feeding termites as compared to their wood-feeding counterpart, reflecting the higher methane emission rates of soil-feeding termites (Brune, 2018, 2019).

\section{The carbohydrate-active enzymes of termite gut prokaryotes}

We investigated the evolution of prokaryotic carbohydrate-active enzymes (hereafter: CAZymes) using the same 129 gut metagenome assemblies used to investigate gut prokaryotic composition. The de novo assemblies of these 129 gut metagenomes contained an average of 127,159 prokaryotic open reading frames (ORF). We identified ORFs coding for CAZymes using Hidden Markov model searches against the dbCAN2 database (Zhang et al., 2018). As a first step, we investigated the evolution of enzymes derived from prokaryotes with no consideration of their taxonomic origin. In total, we found 346 CAZyme categories in 129 gut metagenomes that 
consisted of 205 glycoside hydrolases (GHs), 57 glycoside transferases (GTs), 18 enzymes with carbohydrate-binding modules (CBMs), 16 carbohydrate esterases (CEs), 41 polysaccharide lyases (PLs), and 9 redox enzymes with auxiliary activities (AAs) (Table S5). We did not find any CAZymes in only one gut metagenome (that of Araujotermes parvellus, at e-value cut-off below e-30). For the other 128 gut metagenomes, the number of CAZyme categories varied between 5 and 139 per gut metagenome. Five GH families, GH2, GH3, GH10, GH31, and GH77, were found in more than $85 \%$ of the termite species. $14 \mathrm{GHs}$, seven of which had putative lignocellulolytic activity, were found in 75 to $85 \%$ of the termite species. Therefore, glycoside hydrolases previously found to be abundant in the gut of particular termite species (e.g. Warnecke et al., 2007, Calusinska et al., 2020) are universally part of the gut enzymatic repertoire of termites.

We calculated the Moran I index on the abundance of 211 CAZymes, including 146 CAZyme families and 65 sub-families, present in more than $10 \%$ of termite species, and found an autocorrelation signal with the termite phylogenetic tree for 107 CAZymes. The autocorrelation signal remained significant after FDR correction for 77 CAZymes (Figure 2, Table S6). Therefore, as for gut prokaryotic composition, termite phylogeny is predictive of the CAZyme repertoire present in termite guts.

Two factors that potentially affect the prokaryotic CAZyme repertoire of termite gut prokaryotes are diet and co-occurring non-prokaryotic cellulolytic symbiotic partners. We distinguished four termite groups: soil-feeding Termitidae (SF) and wood-feeding Termitidae excluding Macrotermitinae (WF), which host no other symbionts than gut prokaryotes (Brune, 2014), the fungus-cultivating Macrotermitinae (FC), which feed on wood or plant litter and cultivate cellulolytic fungi of the genus Termitomyces (Rouland-Lefèvre, 2000), and lower termites (LT), which feed on wood and host cellulolytic flagellates in their gut (Inoue et al., 2000). Overall, the abundance of prokaryotic CAZymes was the highest in WF and the lowest in SF, while LT and FC fell between these two extremes (Table S7). This is consistent with the scarcity of lignocellulose in the diet of SF, which predominantly feed on the nitrogen-rich fraction of the soil, including microbial biomass and organic residues associated with clay particles (Ji and Brune, 2001, 2005; Ngugi et al., 2011; Ngugi and Brune, 2012). The intermediate abundance of prokaryotic CAZymes in FC and LT reflects their dependence on Termitomyces fungi for 
lignocellulose digestion (Poulsen et al., 2014) and on gut flagellates that encode for diverse cellulolytic enzymes (Yamin, 1981; Nishimura et al., 2020), respectively.

Task partitioning between gut prokaryotes and other symbionts -in which both partners participate in different steps of wood digestion and provide different sets of CAZymes- could be revealed from the gut metagenomes of LT and FC. Principal component analysis revealed that the prokaryotic CAZyme repertoire differs considerably among SF, LT, FC, and WF (Figure 3A). To characterize more accurately the contribution of termite gut prokaryotes to wood digestion, whenever possible, we identified the substrate of each 211 CAZymes (including 146 families and 65 subfamilies) present in more than $10 \%$ of termite species. We individually compared the abundance of these 211 CAZymes using phylogenetic ANOVA. We found that 178 comparisons were significantly different, and 177 comparisons remained significant after FDR corrections (Figure 3A, Table S7). Notably, we found that the combined seven GHs exclusively identified as cellulases were significantly depleted in LT as compared to other termite groups and were significantly depleted in FC and SF as compared to WF (Figure 2, Table S7). A similar pattern was found for the combined $29 \mathrm{GHs}$ exclusively identified as hemicellulases, which were significantly more abundant in WF than in other termite groups (Figure 3A, Table S7). Therefore, the gut metagenomes of LT and FC appear to be depleted in prokaryotic GHs targeting cellulose as compared to WF, possibly reflecting task partitioning between termite gut prokaryotes and eukaryotic symbionts such as cellulolytic flagellates in LT and Termitomyces in FC. Task partitioning between gut prokaryotes and Termitomyces in FC was previously suggested for Macrotermes natalensis (Poulsen et al., 2014), with gut symbionts primarily participating to the final digestion of oligosaccharides and Termitomyces performing the breakdown of complex carbohydrates. In support of this hypothesis, several GHs, such as GH8, GH26, GH45, GH5_2, and GH53, largely depleted from the gut metagenomes of LT were highly expressed by the gut cellulolytic flagellates of $C$. formosanus (Nishimura et al., 2020), and were abundant in the gut metagenomes of WF. However, several GHs encoded by gut prokaryotes are also highly expressed by the gut cellulolytic flagellates of C. formosanus (e.g. GH13_8, GH36, GH3, GH92, GH133) (Nishimura et al., 2020). The extant of the complementarity between the CAZyme repertoires of gut flagellates and prokaryotes is therefore unclear and requires further investigation. 
We next investigated the taxonomic origin of the prokaryotic CAZymes found in the same 129 whole gut metagenomes. We focused on the $19 \mathrm{GHs}$ found in more than $10 \%$ of termite species and embedded in contigs longer than $5000 \mathrm{bps}$, allowing taxonomic annotation based on several genes. Contigs including genes with discordant taxonomic annotations potentially indicate horizontal gene transfers, as is common among bacteria (Ochman et al., 2000), and were removed. We found that Bacteroidota were a significant source of GH2, GH9, GH10, GH20, GH28, GH29 ,GH30, GH31, and GH130 in FC and LT, while, as previously described (Marynowska et al., 2020), they rarely encoded these GHs in non-Macrotermitinae Termitidae (WF and SF) (Figure 4, Table S8). In contrast, Fibrobacteres, which were very rare in LT, were a significant source of GH2, GH3, GH8, GH9, GH10, GH11, GH18, GH26, GH30, GH43, GH94, and GH130 in WF. Two other bacterial phyla, Spirochaetota and Firmicutes A, encoded most of the investigated GHs and were important contributors of GHs in WF (Figure 4, Table S8). Therefore, the primary contributors of GHs are distinct between lower and higher termites. These results are consistent with previous reports indicating a possible involvement of the ectosymbiotic Bacteroidota of some oxymonadid flagellates in cellulose and hemicellulose hydrolysis (Yuki et al., 2015; Treitli et al., 2019) in lower termites, while Fibrobacteres, Spirochaeota, and/or Firmicutes are major agents in cellulose and hemicellulose degradation in higher termites (Warneke et al., 2007; He et al., 2013; Tokuda et al., 2018; Calusinska et al., 2020; Marynowska et al., 2020). Our comprehensive analyses strongly indicate that the loss of cellulolytic flagellates in the ancestor of higher termites was accompanied by a major reworking of the cellulolytic bacterial communities, from Bacteroidota in LT to Fibrobacterota and Spirochaeota in WF and to Firmicutes in SF.

CAZymes are often organized as polysaccharide utilization loci (PULs) that target complex polysaccharides (Terrapon et al., 2015). To search for PULs in our metagenomes, we reconstructed metagenome-assembled genomes (MAGs) by grouping contigs with similarities in sequence composition and depth of coverage. In total, we obtained 654 prokaryotic MAGs that ranged in completeness from $30 \%$ to $100 \%$ with $<10 \%$ contamination for lineage-specific marker genes. We kept low quality MAGs, with completeness between $30 \%$ to $50 \%$, as several such MAGs possessed complete pathways of interest (Figure S3, Table S9). The 654 MAGs included members of 16 phyla of bacteria and four phyla of archaea and included representatives of all major prokaryote phyla known to be present in termite gut. We found 128 PULs distributed across 130 MAGs, including 31 MAGs of Bacteroidota, 71 MAGs of Firmicutes, 13 MAGs of Proteobacteria, 12 
MAGs of Spirochaetota, two MAGs of Actinobacteria, and one MAG of Verrucomicrobiota (Table S10). Sixteen PULs, found in 10 MAGs, had all the PUL components and mainly targeted lignocellulose components such as cellulose and xylan, and saccharides such as melibiose, alignate, and lactose. 107 PULs found in 74 MAGs encoded for more than one substrate but did not have all the PUL components, possibly reflecting the incompleteness of our MAGs or missing components nonessential for their activity, as experimentally demonstrated in the xylan utilization system (Xus) of a Bacteroidota associated with Pseudacanthotermes (Wu, 2018). Altogether, our data provide an overview of the PUL distribution in termite gut microbes.

\section{Reductive acetogenesis in termite gut}

The fermentation of wood fibers by the termite gut microbiota produces mostly acetate, which is used by the termite host, but also $\mathrm{H}_{2}$ and $\mathrm{CO}_{2}$ (Hungate, 1939; Brune, 2014). Most of the $\mathrm{H}_{2}$ is used to produce additional acetate by the reduction of $\mathrm{CO}_{2}$ (Breznak and Switzer, 1986; Brauman et al., 1992; Pester and Brune, 2007). We focused on the genes of seven enzymes of the WoodLjungdahl pathway (WLP) of reductive acetogenesis that are present in all acetogens from termite guts identified to date, namely formate dehydrogenase $\mathrm{H}(f d h F)$, formate-tetrahydrofolate ligase

(fhs), methylenetetrahydrofolate dehydrogenase (folD), 5,10-methylenetetrahydrofolate reductase $(m e t F)$, acetyl-CoA synthase (acsABCDE), phosphotransacetylase (pta), and acetate kinase (ack), which are essential to operate the bacterial WLP (Schuchmann and Müller, 2014). We compared the relative abundance of these markers across the 129 whole gut metagenomes used for previous analyses and found a significant phylogenetic autocorrelation signal with the termite phylogenetic tree for five of the seven enzymes, two of which remain significant after FDR correction ( $f d h F$ and acsABCDE) (Figure 5, Table S11). Together with the five other enzymes, which also occur in many other bacteria, the simultaneous presence of $f d h F$ and $a c s A B C D E$ is a strong predictor for the distribution of reductive acetogenesis across the termite phylogenetic tree.

The seven enzymes encoded by all acetogens significantly differed in relative abundance among the four termite groups. They were generally more abundant in LT and WF than in FC and SF (Figure 3B, Table S11). These analyses are in agreement with previous studies that measured the potential rates of acetogenesis in a smaller set of termite species, and corroborate the hypothesis that reductive acetogenesis is mostly associated with a diet of wood and is less important in fungus- 
cultivating Macrotermitinae and in soil-feeding lineages (Brauman et al., 1992; Tholen and Brune, 1999).

To determine the identity of the acetogens, we searched each MAG for the genes of the seven enzymes associated with reductive acetogenesis. We found 44 MAGs associated with six termite families and Cryptocercus that encoded at least five of the seven enzymes, but none of these MAGs contained the complete set of genes (Table S12, Figure 6A). In addition to formate dehydrogenase $\mathrm{H}(f d h F)$, we also searched for the genes encoding [FeFe] hydrogenase Group A4 (HydA) and the iron-sulfur cluster proteins $(\mathrm{HycB3}, \mathrm{HycB} 4)$, the other subunits of the hydrogen-dependent $\mathrm{CO}_{2}$ reductase (HDCR) complex catalyzing the first step of $\mathrm{CO}_{2}$ reduction to formate (Schuchmann and Müller, 2012; Ikeda-Ohtsubo et al., 2016). Two MAGs lacked $f d h F$ but contained all other genes of the WLP and the HDCR complex (Table S12, Figure 6A). These MAGs belonged to the Desulfobacterota family Adiutricaceae, which comprises the putatively acetogenic Candidatus Adiutrix intracellularis, a flagellate endosymbiont from the archotermopsid Zootermopsis, and numerous uncharacterized representatives from other lower and higher termites (Ikeda-Ohtsubo et $a l .$, 2016). Like $C a$. Adiutrix intracellularis, none of the four MAGs encoded a sulfate reduction pathway. They were found in the rhinotermitid Dolichorhinotermes and in the higher termite Microcerotermes, indicating that the putatively free-living members of Adiutricaceae from higher termites (which lack gut flagellates) are also acetogenic.

Because none of the other MAGs encoded a complete WLP, we could not unambiguously attribute acetogenic status to any other prokaryote lineage. Considering the high rates of reductive acetogenesis in many lower and higher termites, particularly the wood-feeding species (Brauman et al., 1992), this may be explained either by the incompleteness of our MAGs or the failure to assemble any genomes of the populations responsible for the acetogenic activity. Based on the low free energy yields of both reductive acetogenesis and methanogenesis, it has been speculated that the proportion of (hydrogenotrophic) acetogens among the prokaryotic community in termite hindguts may be as low as that of (hydrogenotrophic) methanogens (Loh et al., 2021). The problem of genome assembly from low abundance populations would be exacerbated by a high species diversity among members of a particular metabolic guild. Alternatively, the absence of a complete reductive acetogenesis pathway among our MAGs may be genuine. This could be the case among the MAGs assigned to the family Treponemataceae B. Although the first isolate of this lineage is 
a homoacetogen with a complete WLP (Leadbetter et al., 1999), none of the other species isolated to date are acetogenic (Song et al., 2021). With the exception of Treponema primitia (Graber et al., 2004), Candidatus Treponema intracellularis (Ohkuma et al., 2015), and Candidatus Adiutrix (Ikeda-Ohtsubo et al., 2016), the identity of the populations responsible for reductive acetogenesis in termite guts, including the putatively acetogenic Candidatus Termitimicrobium (Bathyarcheia; Loh et al., 2021) remains open to speculation.

\section{Methanogenesis in termite gut}

The methanogenic archaea present in the gut of termites consume a large fraction of $\mathrm{H}_{2}$ and are responsible for 3\% of global methane emissions (Brune, 2018, 2019). We searched the 129 gut metagenomes used in earlier analyses for genes that are part of methanogenesis pathways. Because of the low abundance of Archaea in termite guts (Brune 2019; Loh et al., 2021), the abundance of genes involved in methanogenesis was often near, or below, our detection threshold. As a consequence, we were unable to analyze each gene independently, but instead calculated the Moran's I index using the abundance of genes encoding the methyl-coenzyme $\mathrm{M}$ reductase complex (mcrABG), which catalyzes the final step of methanogenesis (Evans et al., 2019), and found no autocorrelation signal with the termite phylogenetic tree (Figure 5, Table S11).

We compared the abundance of $m c r A B G$ among the four termite groups and found no significant differences (Figure 3B, Table S11). However, this lack of significance probably reflects the low abundance of archaeal reads in our assemblies, rather than an actual uniformity of methanogenesis pathways across termites, as methane emission rates are known to be diet-related and particularly high in species feeding on soil (e.g., Brauman et al., 1992; Bignell and Eggleton, 1995; Bignell et al., 1997; Sugimoto et al., 1998).

We searched our gut metagenomes for operons encoding $\operatorname{mcr} A B G$, and found 14 operons, belonging to four methanogenic archaeal orders, Methanomassiliicoccales, Methanobacteriales, Methanomicrobiales, and Methanosarcinales, derived from the gut metagenomes of 14 termite species, including four of the eight families of LT, and five of the nine subfamilies of Termitidae (Table S13). All mcrABG operons of LT were classified to Methanobacteriales, which is in agreement with previous reports on the prevalence of Methanobacteriales in LT (Brune, 2019). 
An exception was found in the gut metagenome of Porotermes quadricollis, which yielded an mcrABG operon from Methanomethylophilaceae (order Methanomassiliicoccales). This is unusual, because members of this order are frequently encountered in higher termites and millipedes (Paul et al., 2012) but have been detected only once in the lower termite Reticulitermes speratus (Shinzato et al., 2001).

Next, we analyzed the methanogenic capacities of 26 MAGs of Archaea reconstructed from the gut metagenomes of 23 termite species from four termite families and the cockroach Cryptocercus. Only 13 MAGs belonging to Methanomicrobiales, Methanobacteriales, Methanosarcinales, and Methanomassiliicoccales encoded the mcrABG complex, indicating that the assemblies are incomplete (Figure 6B, Table S14). Five of these 13 MAGs possessed complete pathways for methylotrophic methanogenesis and one MAG possessed complete pathways for hydrogenotrophic methanogenesis (Figure 6B). The five MAGs showing genomic evidence of methylotrophic methanogenesis included one MAG of Methanosarcinales (genus Methanimicrococcus) and four MAGs of Methanomassiliicoccales, including three MAGs classified to genus Methanoplasma and one MAG classified to family Methanomethylophilaceae. Only two MAGs of Methanoplasma encoded a methanol:coenzyme M methyltransferase $(m t a A B C)$ complex, which is required for growth on methanol and typical for all members of this lineage (Lang et al., 2015), and only one of the MAG of Methanosarcinales and one MAG of Methanoplasma encoded a complete heterodisulfide reductase complex (HdrA2B2C2/mvhADG) present in most methanogens (Thauer et al., 2008; Buckel and Thauer, 2013), underscoring the incompleteness of the MAGs. The same was true for hydrogenotrophic methanogenesis, for which only one MAG belonging to Methanobacteriaceae (genus Methanobrevibacter $C$ ) possessed most of the genes required for the reduction of $\mathrm{CO}_{2}$ to methane, including a heterodisulfide reductase $(H d r A B C / m v h A D G)$ complex, an iron-sulfur flavoprotein along with a F420-independent hydrogenase $(F d h)$, and a F420 reducing hydrogenase $(F r h A B C$ ) (Figure 6B, Table S14). The absence of aceticlastic methanogens is in agreement with previous reports (Brune, 2018, 2019). Overall, our results highlight the diversity of methanogens found in termite guts, and the diversity of the pathways they use.

\section{Sulfate-reducing prokaryotes}


Sulfate-reducing bacteria are potential $\mathrm{H}_{2}$-consumers in the gut of termites (Brauman et al., 1990; Kuhnigk et al., 1996; Dröge et al., 2005) (Figure 5). However, sulfate concentration is low in termite gut, as is $\mathrm{H}_{2}$ consumption by sulfate-reducing bacteria (Dröge et al., 2005; Brune and Ohkuma, 2011). We found all the genes of the dissimilatory sulfate reduction pathway, namely, the two subunits of adenylylsulfate reductase ( $\operatorname{pprA}$ and $a p r B)$, sulfate adenylyltransferase (sat), and dissimilatory sulfite reductase $(\operatorname{ds} r A B)$, in six out of eight lower termite families, all the higher termite subfamilies, and Cryptocercus. The abundance of aprAB and sat were significantly correlated with the termite phylogenetic tree, and the correlation remained significant after FDR correction for sat (Figure 5, Table S11).

Comparisons of the four termite groups showed that the abundance of $a p r A B$ was significantly higher in WF than in SF and the abundance of sat was significantly higher in LT than WF and SF (Figure 3B, Table S11). While sulfate reducers have been isolated from the guts of LT, FC and SF (Brauman et al., 1990; Kuhnigk et al., 1996), we found metagenomic evidence that sulfate reduction is also prevalent in WF.

Next, we analyzed the sulfate-reducing capabilities of our 654 MAGs and found a complete pathway for dissimilatory sulfate reduction in four MAGs (Figure 6C, Table S15). Three of these MAGs, found in the termites Parrhinotermes, Reticulitermes, and Tumulitermes, were assigned to Desulfovibrionaceae (Desulfobacterota), which are common in the termite gut and generate energy via sulfate respiration (Sato et al., 2009; Kuwahara et al., 2017). Of note, the fourth MAG, retrieved from the gut metagenome of the apicotermitine Heimitermes laticeps, belonged to the Proteobacteria family Burkholderiaceae, a bacterial family that was, prior to this study, largely unreported from termite guts, and that is abundant in Apicotermitinae and in the termite clade that includes the Cubitermitinae, the Pericapritermes-group, and the Termes-group. The evidence for dissimilatory sulfate reduction in Burkholderiaceae termite guts suggest that the capacity for sulfate respiration is more widely distributed than expected.

\section{Nitrogen recycling by termite gut prokaryotes}

Because the content of nitrogen in wood is low, termites have evolved mechanisms of nitrogen conservation. The termite gut microbiota contributes to the nitrogen metabolism of its host by 
recycling nitrogen (Breznak, 2000; Hongoh, 2011). Like most insects, termites convert waste products from nitrogen metabolism into uric acid, but, unlike other insects, the gut prokaryotes of termites degrade uric acid into ammonia, which is subsequently assimilated by the gut microbiota (Brune, 2014). We searched the 129 metagenomes used for previous analyses and found only few genes possibly involved in uric acid degradation, including 11 aegA (a putative oxidoreductase suspected to be involved in uric acid degradation by Enterobacteriaceae (Iwadate and Kato, 2019)) in six termite species. Since the uricolytic prokaryotes isolated from termite guts are strict anaerobes (Potrikus and Breznak, 1980, 1981; Thong-On et al., 2012), it is likely that they use alternative, so far unknown, pathways. Termite tissues reportedly lack uricase activity (Potrikus and Breznak, 1981), but when we examined the transcriptomes of 53 termite species generated by Buček et al. (2019), we found evidence for the expression of a gene encoding urate oxidase in 20 termite species belonging to four termite families (Figure S4). This indicates that termites should be able to carry out the first step of uric acid degradation. However, the extent of the contribution of the termite host to uricolysis and the identity of the uricolytic prokaryotes and their catabolic pathways remain unknown.

The metagenomes of all termite families included numerous prokaryotic genes from other pathways involved in the production of ammonia (Figure 5, Table S11), including ureases (ureABC), which degrade urea into ammonia (Hongoh and Ohkuma, 2010; Ohkuma et al., 2015), and some of the genes of the dissimilatory nitrate reduction pathway (narGHI, napAB, nrfAB), which convert nitrate into ammonia. Among those, the abundance of $u r e A B C$ genes significantly correlated with the termite phylogenetic tree after FDR correction (Figure 5, Table S11). We also found in the metagenomes of all termite families genes from pathways involved in amino acid biosynthesis from ammonia, including glutamine synthetase $(g \ln A)$ and glutamate synthase $(g l t B D)$, the genes involved in the synthesis of glutamate from ammonia, and carbamate kinase $(\operatorname{arc} C)$, ornithine carbamoyltransferase $(\arg F)$, argininosuccinate synthase $(\arg G)$ and argininosuccinate lyase $(\operatorname{argH})$, the genes involved in arginine biosynthesis from ammonia (Yan 2007). The abundance of $g l t B D$ correlated with the termite phylogenetic tree after FDR correction (Figure 5, Table S11). Therefore, the termite phylogeny is a good predictor of the enteric abundance of some of the prokaryotic genes involved in ammonia metabolism in termites. 
We compared the four termite groups using the relative abundance of the nitrogen-recycling genes and found that the abundance of $u r e A B C$ differed among termite groups, with the gut metagenomes of LT and WF significantly enriched in ureABC as compared to those of SF and FC (Figure 3B, Table S11). In contrast, the abundance of some of the genes of the dissimilatory nitrate reduction pathway, such as $n a p A B$ and narGHI, was significantly reduced in the gut metagenomes of WF compared to SF and FC (Figure 3B, Table S11). This suggests that the high rates of nitrate ammonification previously found in two soil-feeding species (Ngugi and Brune, 2012) is a characteristic that all soil-feeding termites share with fungus-cultivating termites. We also found that $g l t B D$ was significantly enriched in LT as compared to other termite groups, while $\operatorname{argFGH}$ was significantly enriched in LT and WF as compared to SF (Figure 3B, Table S11). The low abundance of genes involved in ammonia assimilation in soil-feeding termites is likely linked to their diet, which includes soil peptidic residues (Ji and Brune, 2001, 2005).

Next, we searched our 654 MAGs to determine the taxonomic identity of the prokaryotes involved in nitrogen recycling. Six MAGs possessed the three ureases $u r e A B C$, thence encoded enzymes to convert urea into ammonia, and 15 MAGs included a complete dissimilatory nitrate reduction pathway that convert nitrate into ammonia. All these MAGs belonged to diverse lineages of Proteobacteria and Campylobacterota (order Campylobacterales), except for one MAG of Firmicutes (genus Bacillus) found in Foraminitermes rhinoceros and endowed with ureABC, narGHI and nirBD (Figure 7A, Table S16). We also found numerous MAGs capable of ammonia assimilation into glutamate and arginine, indicating that ammonia is an important source of nitrogen for many termite gut prokaryotes. 91 MAGs possessed $g \ln A$ and $g l t B D$ for glutamate biosynthesis from ammonia, while 26 MAGs possessed the four genes $\operatorname{arc} C, \arg F, \arg G$, and $\operatorname{argH}$ for arginine biosynthesis from ammonia via the urea cycle, including 12 MAGs that also contained the glutamate biosynthesis pathway (Figure 7A, Table S16). 66 MAGs encoding glutamate biosynthesis genes, and 15 MAGs with arginine biosynthesis genes, also possessed the ammonium transporter Amt. These MAGs belonged to ten phyla, including 19 MAGs of Proteobacteria from six families, 18 MAGs of Bacteroidota, of which eight belonged to the family Azobacteroidaceae, ten MAGs of Actinobacteria of three families, eight MAGs of the Spirochaetes family Treponemataceae B, eight MAGs of Firmicutes, six MAGs of Campylobacterota, five MAGs of Firmicutes A, three MAGs of Planctomycetota, three MAGs of 
Desulfobacterota, and one MAG of Verrucomicrobia (Figure 7A, Table S16). Therefore, a great many bacterial lineages contribute to the nitrogen metabolism of their termite hosts.

\section{Nitrogen fixation by termite gut prokaryotes}

Many species of wood-feeding termites host dinitrogen-fixing prokaryotes in their gut, which compensate for the low nitrogen content of wood (Breznak, 2000). They fix nitrogen with either the molybdenum-dependent (Nif), vanadium-dependent (Vnf), or iron-only alternative nitrogenases (Anf) (Ohkuma et al., 1999; Yamada et al., 2007; Inoue et al. 2015). We found gene homologs for the structural subunits of these nitrogenases (collectively referred to as nifDHK) in all metagenomes of termite families and in Cryptocercus (Figure 5). Their abundance significantly correlated with the termite phylogeny after FDR correction (Figure 5, Table S11), as was the case for several other pathways involved in nitrogen economy. There were significant differences among termite groups, with the nitrogenase reads in the gut metagenomes of non-FC wood-feeders (LT and WF) being 24.4-fold more abundant than in SF and 20.2-fold more abundant than in FC (Figures 3B, 5, Table S11). This is in line with the higher rate of $\mathrm{N}_{2}$ fixation measured in LT and WF than in SF and FC (Yamada et al., 2007), and reflects the high amount of nitrogen present in soil and fungi, making the energy-demanding process of $\mathrm{N}_{2}$ fixation unnecessary (Brune and Ohkuma, 2011; Hongoh, 2011).

To identify the diazotrophs present in the gut of termites, we taxonomically classified contigs longer than 5000 bps that contained the six genes present in all diazotrophs, nifDHK (which encode nitrogenase), and nifB, nifE, nifN, which encode proteins involved in nitrogenase biosynthesis (Dos Santos et al., 2012). We identified 15 contigs matching these criteria in the gut metagenomes of 12 termite species, representing five of the nine termite families (Table S17). These contigs were assigned to diverse prokaryote lineages, including nine contigs of diverse Bacteroidota, three contigs of the Spirochaetota order Treponematales, two contigs of Proteobacteria family Enterobacteriaceae, and one contig of the archaeal genus Methanobrevibacter. We carried out the same analyses on our MAGs and found 18 MAGs that contained a nifHDKBEN cluster, including seven MAGs that belonged to phyla not represented in the contigs $>5000 \mathrm{bps}$. Among these seven MAGs, there were four MAGs of the Actinobacteriota family UBA8131, one MAG of the Planctomycetota family Thermoguttaceae, one MAG of the Verrucomicrobiota family 
Chthoniobacteraceae, and one MAG of the Firmicutes C order Acidaminococcales (Figure 7A, Table S16). Therefore, the taxonomy of diazotrophs found in our termite species set corroborates previous evidence that termites host diverse communities of diazotrophs in their guts (Ohkuma et al., 2001; Yamada et al., 2007; Desai and Brune, 2012).

We next investigated the taxonomic distribution of diazotrophs across termites. We focused on contigs longer than 5000 bps that included genes with concordant taxonomic annotation and that contained a nifHDK operon (Figure 7B, Table S18). In lower termites, the dominant diazotroph was an undescribed Bacteroidota allied to an ectosymbiont of the Cryptocercus gut flagellate Barbulanympha (Tai et al., 2016). This undescribed Bacteroidota was found in three of the eight families of LT. It was also largely absent from the gut metagenomes of Coptotermes and Heterotermes, which harbor the flagellate endosymbiont Candidatus Azobacteroides as the main diazotroph (Hongoh et al., 2008). The diazotrophs of Termitidae belonged to various phyla. Notably, we found the $\mathrm{N}_{2}$-fixing Candidatus Azobacteroides in the nasutitermitine Coatitermes (which lacks gut flagellates), and a $\mathrm{N}_{2}$-fixing Treponematales in Mastotermes, highlighting that the dominant lineages of diazotrophs in particular termite lineages are also harbored at a low abundance by unrelated species of termites (Figure 7B, Table S18). Therefore, our results indicate that the phylogenetic position of termite species determined, at least partly, the taxonomy of their dominant diazotrophs.

\section{CONCLUSION}

The metagenomics and metatranscriptomics surveys of termite guts carried out so far targeted a limited number of termite species (e.g. Warnecke et al., 2007; He et al., 2013; Liu et al., 2018; Tokuda et al., 2018; Marynowska et al., 2020), and thus did not permit an investigation of how the gut microbiome of these social roaches has been evolving in term of function and composition since termite origin, $\sim 150$ Million years ago. To address this issue, and to provide a global picture of the taxonomic and functional composition of the termite gut microbiome, we generated gut metagenomes for a comprehensive set of 145 termite species. The analyses of this dataset revealed that: (1) gut prokaryotic genes involved in the main nutritional functions are generally present across termites, suggesting these genes were already harbored by the common ancestor of modern termites; (2) the termite phylogenetic tree is largely predictive of the gut bacterial community 
composition and the nutritional function they exert; and (3) the acquisition of a diet of soil was accompanied by a change in the stoichiometry of genes and metabolic pathways involved in important nutritional functions rather than by the acquisition of new genes and pathways.

The analyses of our 146 gut metagenomes indicated that prokaryotic CAZymes, genes of the reductive acetogenesis, sulfur reduction, and methanogenesis pathways, and genes involved in nitrogen fixation and recycling, are present across the nine termite families. Therefore, the nutritional functions previously known to be performed by the gut prokaryotic symbionts of particular termite species (e.g. Warnecke et al., 2007; Calusinska et al., 2020) are performed in the gut of all termites. These results strongly suggest that the gut prokaryotes performing important nutritional functions were already harbored by the common ancestor of modern termites. Following this scenario, the ancestor of termites did not only acquire their charismatic gut cellulolytic flagellates (Nalepa, 1991), but also acquired several bacterial and archaeal lineages that make up a sizable fraction of the gut microbiota of modern termite species. In support of this hypothesis, many termite gut bacteria phylotypes form monophyletic groups present in the gut of various termite families and distantly related to bacteria found in other environments, such as in the guts of other animals, including cockroaches (Bourguignon et al., 2018). Therefore, as the cockroach-like ancestor of termites evolved wood-feeding, it is likely that it recruited facultative gut microbes able to degrade wood and participate in the nitrogen economy as essential gut symbionts.

Our analyses indicate that the phylogenetic position of termite species is partly predictive of the functions of gut bacterial communities. This is best illustrated by CAZymes whose abundance often correlated with the termite phylogenetic tree. Correlation with the termite phylogenetic tree, however, was not found for some genes, such as the $\operatorname{mcr} A B G$ genes of the methanogenesis pathway, the genes of sulfate reduction pathway, and the genes of the dissimilatory nitrate reduction pathway. Whether this lack of correlation is genuine, or whether it reflects insufficient depth of sequencing, is unclear and requires further study. In any case, our results indicate that the correlation found between the phylogenetic tree of termites and their gut bacterial and protist communities (Rahman et al., 2015; Tai et al., 2015) are also found for some gut microbial functions.

The comparison of four termite groups, soil-feeding Termitidae (SF), fungus-cultivating Macrotermitinae (FC), non-Macrotermitinae wood-feeding Termitidae (WF), and lower termites 
(LT), reveals that genes and metabolic pathways important to termites are present in all termite species, but their abundances vary among groups. Notably, the gut metagenomes of SF possessed on average fewer CAZymes, nitrogenases, reductive acetogenesis, and sulfate-reducing genes than the gut metagenomes of other termite groups. Therefore, as pointed out by Marynowska et al. (2020), the gut prokaryote communities of SF retain important carbohydrate metabolism capabilities. Nevertheless, our dataset clearly indicate that these abilities are much reduced in soilfeeders compared to wood-feeders. Overall, our results support the idea that the acquisition of soilfeeding was accompanied by changes in the abundance of the gut prokaryote metabolic pathways important to termite nutrition.

\section{ACKNOWLEDGMENTS}

We thank the DNA Sequencing Section and the Scientific Computation and Data Analysis Section of the Okinawa Institute of Science and Technology Graduate University, Okinawa, Japan, for assistance with sequencing and for providing access to the OIST computing cluster, respectively.

\section{FUNDING}

This work was supported by the subsidiary funding to OIST, by the Czech Science Foundation (project No. 20-20548S), by the Internal Grant Agency of the Faculty of Tropical AgriSciences, CULS (20213112), by the Australian Research Council through a Future Fellowship to NL, and by the Japan Society for the Promotion of Science through a Kakenhi grant to GT (17H01510) and a DC2 graduate student fellowship awarded to JA.

\section{AUTHORS' CONTRIBUTIONS}

J.A., Y.K., and T.B. conceptualized the experiments and approach. J.S., P.S. Y.R., Y.C.P., K.Y.K., D.S.D., G.T., and T.B. collected the samples. J.A and C.C performed the lab experiments. J.A., Y.K., and T.B. designed the data analyses. J.A. and Y.K. performed the bioinformatics analyses. A. Bucek examined the urate oxidase function in termite transcriptomes. J.A. and T.B. wrote the 
paper, with significant contribution from A. Brune, V.H., and G.T. All authors read and accepted the final version of this manuscript.

\section{COMPETING INTERESTS}

The authors declare that they have no competing interests.

\section{CONSENT FOR PUBLICATION}

Not applicable.

\section{DATA AVAILABILITY}

Raw sequence data generated in this study have been deposited on MG-RAST (https://www.mgrast.org/mgmain.html?mgpage=project\&project=mgp100619) (see Table S1 for individual IDs). MAGs generated in this study are available on Figshare (https://doi.org/10.6084/m9.figshare.17031674.v1). The scripts used in this study are available on github (https://github.com/oist/EGU-The-functional-evolution-of-termite-gut-microbiota).

\section{ETHIC APPROVAL}

Not applicable.

\section{METHODS}

\section{Sample collection}

We collected a total 145 termite samples and one sample of the cockroach Cryptocercus kyebangensis (Table S1, Figure S1). These samples were representative of the global termite diversity. All samples were preserved in RNA-later ${ }^{\circledR}$ and stored at $-80^{\circ} \mathrm{C}$ until DNA extraction. 


\section{DNA extraction and sequencing}

Genomic DNA extraction was performed on the whole guts of five workers using the NucleoSpin Soil kit (Macherey-Nagel) according to manufacturer's protocol. Library preparation was performed using the KAPA Hyperplus Kit, which is based on a unique dual tag indexing approach that minimizes the effects of index hopping. Libraries were either PE250-sequenced on the Illumina HiSeq2500 platform or PE150-sequenced on the Illumina HiSeq4000 platform (Table S1).

\section{Data filtering and assembly of metagenomic reads}

Raw reads were filtered based on their quality. Reads with average Phred quality score below 30 were removed using Trimmomatic v 0.33 (Bolger et al., 2014). The "SLIDINGWINDOW" was set to "4:30" to trim low quality bases (Phred quality score below 30) from the 3 ' end of the reads. We removed the 16 base pairs at the 5' end of each read using the "HEADCROP" option because we observed over-represented k-mers in this region of the reads. Reads shorter than 50bps were removed.

The quality-controlled reads were assembled into contigs using SPAdes v 3.11.1 (Nurk et al., 2017) with the "meta" option and k-mer sizes of $21,31,41,51,71$. The assembly quality was checked using the "metaquast" option of QUAST v 3.1 (Quality Assessment for Genome Assemblies) based on weighted median contig size (N50) (Gurevich et al., 2013) and percent of reads mapped to the contigs (Langmead et al., 2012; Papudeshi et al., 2017). Only the reads mapped to prokaryotic contigs were examined in this study (see the taxonomic annotation and functional annotation sections below).

\section{Termite phylogenetic tree reconstruction}

We build a phylogenetic tree of termites using mitochondrial genomes retrieved from metagenome assemblies. Mitochondrial contigs derived from termites were identified using BLAST search (sequence length >5000 and percent identity >90) (Altschup et al., 1990) against previously published whole mitochondrial genomes of termites (Bourguignon et al., 2015, 2016, 2017; Wang 
et al., 2019). Mitochondrial genomes were complete, or near-complete, in most cases. Each contig derived from mitochondrial genomes was annotated using the MITOS webserver (Bernt et al., 2013). The 13 protein-coding genes, two rRNA genes, and 22 tRNA genes were aligned with MAFFT v 7.305 (Katoh et al., 2002) using default settings. The alignments were concatenated and the third codon position of protein-coding genes was removed. The dataset was partitioned into four subsets: one for the first codon position of protein-coding genes, one for the second codon position of protein-coding genes, one for the two rRNA genes, and one for the 22 tRNA genes. A Bayesian phylogenetic tree was generated using BEAST v 2.4.8 (Suchard et al., 2018). We used an uncorrelated relaxed lognormal clock model (Drummond et al., 2006), and a Birth Death speciation process as tree prior (Gernhard, 2008). The molecular clock was calibrated using nine fossil calibrations used by Bucek et al., (2019) (Table S19). The fossil calibrations were implemented as exponential priors on node times. Because transcriptome-based phylogenies unambiguously support the monophyly of Sphaerotermitinae and Macrotermitinae (Bucek et al., 2019) (unlike mitochondrial genome-based phylogenies; Bourguignon et al., 2017), we constrained Sphaerotermitinae + Macrotermitinae to be monophyletic. Similarly, we constrained non-Stylotermitidae Neoisoptera to form a monophyletic group. The MCMC chain was sampled every 1000 steps over a total of 0.4 billion generations. The convergence of the chain was assessed using Tracer v 1.7.1 (Rambaut et al., 2018), and the initial 10 percent was discarded. We carried out two replicate MCMC runs to ensure convergence of the chain.

\section{Reconstruction of Metagenome Assembled Genomes}

We reconstructed Metagenome Assembled Genomes (MAGs) from metagenomes contigs using CONCOCT v 0.4 .0 (Alneberg et al., 2014) implemented in the metawrap software v 0.9 (Uritskiy et al. 2018) with default parameters. MAG quality checking, based on 43 single-copy marker genes (Table S9), was performed with CheckM v 1.0.11 (Parks et al., 2015). High-quality MAGs, medium-quality MAGs, and low-quality MAGs with upward of 30\% completeness and downward of $10 \%$ contamination were retained (Table S9) (Bowers et al., 2017). We retained low-quality MAGs that were at least $30 \%$ complete because, in some cases, they were endowed with complete pathways. Despite having fewer single-copy marker genes, $65.35 \%$ of these MAGs possessed more than 10 transfer RNA genes (tRNA) and $17.54 \%$ had at least one of the three ribosomal RNA 
genes (rRNA). All MAGs that did not meet these criteria were discarded. In addition, we discarded MAGs with obvious mismatches among marker genes. To identify these MAGs, we built Maximum Likelihood phylogenetic trees for all 43 single-copy marker genes with FastTree v 2.1.11 (Price et al., 2009). MAGs that fall in different phyla for different marker genes were considered as having obvious mismatches and were discarded. The rRNA genes were extracted using METAXA2 software (Bengtsson-Palme et al., 2015), tRNA genes were predicted via tRNAscan-SE tool (Chan and Lowe, 2019), and MAG coverage was calculated with the "metawrap quant_bins" command of the metawrap software (Uritskiy et al., 2018).

\section{Taxonomic annotation}

The annotation of genomic features of bacterial and archaeal contigs and MAGs was carried out with Prokka v 1.14 (Seemann, 2014). This step allowed the identification of coding sequences (CDS), ribosomal RNAs (rRNA), and transfer RNAs (tRNA), which were used in downstream analyses. To identity the taxonomy of the metagenome contigs, we taxonomically annotated single-copy marker genes and other protein-coding genes in contigs longer than 1000bps. 40 single-copy marker genes were extracted using mOTU software ver1 (Sunagawa et al., 2013; Wu et al., 2013). Single-copy marker genes were taxonomically annotated using DIAMOND BLASTp (Buchfink et al., 2015) with e-value $\leq 1 \mathrm{e}-24$ and output format 102, which uses the lowest common ancestor algorithm for annotation. Other protein-coding genes were annotated using the same settings as marker genes but with DIAMOND BLASTx algorithm. Both annotations were performed using the GTDB ver 95 database as reference (Parks et al., 2020). Taxonomic annotation of MAGs was based on bacterial and archaeal reference trees using GTDB-Tk v1.3.0 based on GTDB ver 95 (Chaumeil et al., 2020).

We used the genomic DNA extracted from whole termite guts to produce 16S rRNA gene PCR amplicon sequences. PCR reactions were carried out using the primer pairs 515F (XXXXXGTGTGYCAGCMGCCGCGGTAA, Parada et al., 2016) and 806R (XXXXXXXXCCGGACTACNVGGGTWTCTAAT, Apprill et al., 2015). All pairs of primers were endowed with unique dual tag indexes ( $8 \mathrm{X}$ overhang on the forward primer and $5 \mathrm{X}$ overhang on the reverse primer) to minimize the effects of index hopping between libraries. We conducted PCR amplifications using Takara Tks Gflex DNA Polymerase with the following conditions: 
initial denaturation $\left(3 \mathrm{~min}\right.$ at $\left.94^{\circ} \mathrm{C}\right), 30$ cycles of amplification $\left(45 \mathrm{~s}\right.$ at $94^{\circ} \mathrm{C}, 60 \mathrm{~s}$ at $50^{\circ} \mathrm{C}$, and 90 $\mathrm{s}$ at $\left.72^{\circ} \mathrm{C}\right)$, and a terminal extension $\left(10 \mathrm{~min}\right.$ at $\left.72^{\circ} \mathrm{C}\right)$. All PCR reactions were scaled down using one half of the reagents recommended in the manufacturers protocol. Prepared libraries were mixed in equimolar concentration and paired-end-sequenced on the Illumina MiSeq platform. The analysis of the $16 \mathrm{~S}$ rRNA gene amplicon sequences was performed with mothur v1.44.1 (Schloss et al., 2009) following the standard procedure for Illumina data analysis described by Kozich et al. (2013). After removing low-quality reads and chimera, sequences were clustered into operational taxonomic units (OTUs) at a sequence similarity level of $97 \%$ using VSEARCH (Rognes et al., 2016). Sequences were classified using the naïve Bayesian classifier (Wang et al., 2007) implemented in mothur and the SILVA reference database release 138 (Quast et al., 2013). The abundance of every family inferred from both 16S rRNA gene amplicon data and metagenomic data was then compared. In total 143 prokaryote lineages received identical family-level annotation in both datasets.

\section{Functional annotation}

We carried out functional annotation of the CDSs identified with Prokka v.1.14.5 (Seemann, 2014) for all contigs and MAGs that were taxonomically annotated as bacteria or archaea using the "metagenome" option. We used the CAZy database (Lombard et al., 2014) as a reference to identify CDSs with carbohydrate metabolizing properties. Protein sequences were searched against a set of profile Hidden Markov models (HMM) representing CAZy domains deposited in the dbCAN database release 7 (Yin et al., 2012). We used an e-value lower than e-30 and coverage greater than 0.35 as thresholds to extract best domain matches.

Hydrogenases were annotated by means of HMM searches against the Pfam database version 32.0 (El-Gebali et al., 2019) using an e-value cut-off of e-30. Catalytic subunits of hydrogenases were classified into different classes using the k-nearest neighbor algorithm implemented in the HydDB webtool (Søndergaard et al., 2016). For the [FeFe] hydrogenase Group A4, we carried out manual inspection of the conserved motifs in the protein sequence (Schuchmann et al., 2018).

We reconstructed prokaryotic metabolic pathways from our metagenomes with KOFam scan v.1.1.0 (Kanehisa et al., 2016; Graham et al. 2018). We used the KEGG database as a reference and e-value cut-off of e-30. Each protein sequence was annotated to gene family level with the 
KEGG-Decode python module (Graham et al., 2018). The MAG metabolic pathways were annotated with KOFam scan v.1.1.0 using default settings. As some MAG gene families appeared to be absent after annotation against the KEGG database, to confirm, or reject, the absence of these gene families, we carried out BLAST searches (Amino acid identity $>60 \%$ and alignment length $>100$ amino acids) against the Annotree protein sequence database (Mendler et al., 2019).

\section{Relative abundance of gene families}

The relative abundance of CDSs was calculated by mapping the raw reads on the sequences. Briefly, the reads were mapped to the assembled contigs annotated as bacteria or archaea. Relative abundance was calculated for each CDS using Salmon v.1.4.0 with the "meta" option. Salmon corrects for GC-content bias, gene-length differences, and sampling effort (Srivastava et al., 2020). Relative abundance of CDSs obtained as Transcripts per Million (TPM) values were retained for downstream analysis if they were embedded into contigs longer than $1000 \mathrm{bps}$ and had more than 1 TPM value. Individual TPM counts were normalized using centered $\log (2)$-ratio (clr) transformation to account for the compositional structure and unequal numbers of reads in our metagenome data. Clr transformation enhances sub-compositional comparisons (gene vs gene, bacteria vs bacteria) and reduces spurious correlations. Positive and negative TPM values indicate positive and negative departure from the overall compositional mean, which is zero (Gloor et al., 2017). Clr transformation of marker genes and functional genes was performed using the $\mathrm{R}$ package propr using 0.65 as a pseudo count to account for zero values (Quinn et al., 2017). We did not calculate TPM for MAGs, but instead used presence/absence to investigate pathway completeness.

\section{Statistical Analysis}

We investigated whether the abundance of the genes and pathways of interest was phylogenetically autocorrelated to the time-calibrated tree of termites. To do so, we calculated the Moran's I phylogenetic autocorrelation index using the R package phylosignal (Keck et al., 2016) on CDSs embedded in contigs longer than 1000 bps and with TPM value higher than 1 . This analysis was carried out for each bacterial and archaeal phylum present in at least 5\% of the metagenomes, 
using the combined 40 single-copy marker genes (see Table S3). A 5\% false discovery rate (FDR) correction was calculated using the p.adjust function implemented in the R package stats (R Core Team, 2014). Similarly, we calculated the Moran's I phylogenetic autocorrelation index for each 211 CAZymes present in more than $10 \%$ of gut metagenomes and carried out a $5 \%$ false discovery rate FDR correction. Finally, the analysis was performed for each gene involved in the reductive acetogenesis, sulfate reducing, nitrogen recycling and nitrogen fixating pathways, and the $m c r A B G$ gene of the methanogenesis pathway combined. We applied a 5\% FDR correction.

To examine whether the abundance of the genes and pathways of interest differed with termite diet and presence of non-prokaryotic co-symbionts, we performed phylogenetic ANOVA using the procD.pgls function implemented in the R package geomorph (Adams and Otárola-Castillo, 2013). A 5\% FDR correction was calculated using the p.adjust function implemented in the R package stats (R Core Team, 2014). Termite diet was determined based on literature data (Donovan et al. 2001, Bourguignon et al. 2011), and was considered as made of wood or soil. Wood-feeding termite species included feeding groups I and II (including grass and leaf litter), while soil-feeding termites included feeding groups III and IV (sensu Donovan et al. 2001). Non-prokaryotic cosymbionts are found in two groups of wood-feeding termites: the lower termites, which include all termites with the exclusion of Termitidae and host cellulolytic flagellates in their gut, and the Macrotermitinae, a subfamily of Termitidae that cultivates cellulolytic Termitomyces in fungal combs. Therefore, we recognized four groups of termites: the lower termites (LT), the soil-feeding termites (all Termitidae, SF), the Macrotermitinae (FC), and the non-Macrotermitinae woodfeeding Termitidae (WF). Similar analysis was performed on prokaryotic lineages encoding CAZyme families present in more than $10 \%$ of termite gut metagenomes in contigs longer than $5000 \mathrm{bps}$, to ensure correct taxonomic annotation. All metagenome contigs longer than 5000 bps with dinitrogen-fixing genes were also examined.

We visualized termite samples according to the abundance of CAZyme families present in their gut metagenomes using Principal Component Analysis (PCA). The PCA was performed using the prcomp function implemented in the R package stats (R Core Team, 2014) and visualized using the R package ggbiplot ( Vu, 2011). Similar analyses were performed on the genes involved in reductive acetogenesis, sulfate reduction, dissimilatory nitrate reduction, urea degradation, 
glutamate biosynthesis, arginine biosynthesis, ammonia transport, nitrogen fixation, and $m c r A B G$ genes of the methanogenesis pathway.

\section{Uricase genes encoded by termites}

We searched the 53 termite transcriptomes previously published by Buček et al. (2019) for the presence of uricases. These transcriptomes were either derived from whole worker bodies or from worker heads, and included species of all termite families. Protein sequences of predicted uricases from termites (XP_023702357, GFG34960), cockroaches (PSN45555, CDO39394), fireflies (KAF529609, XP_031344605), sawflies (XP_015591878, XP_015521616), ant (XP_011159093), fruit fly (NP_476779), and rat (NP_446220) were used as a query in TBLASTn searches. The longest open reading frames for all significant TBLASTn search hits (E-value $<10^{-30}$ ) were identified and translated using hmmer2go obtained from https://github.com/sestaton/HMMER2GO. The nonsense proteins that did not provide any significant BLASTx hit against NCBI RefSeq database (E-value $<10^{-10}$ ) were discarded. The remaining predicted protein sequences, derived from 23 transcripts, were assigned KEGG annotations using eggNOG-Mapper version 4.5 (Huerta-Cepas et al., 2016). The protein sequences were aligned using CLUSTAL W (Larkin et al., 2007) and the alignment was visually inspected.

\section{REFERENCES}

Adams, D.C., and Otárola-Castillo, E. (2013). Geomorph: An R package for the collection and analysis of geometric morphometric shape data. Methods Ecol. Evol. 4, 393-399.

Alneberg, J., Bjarnason, B.S., De Bruijn, I., Schirmer, M., Quick, J., Ijaz, U.Z., Lahti, L., Loman, N.J., Andersson, A.F., and Quince, C. (2014). Binning metagenomic contigs by coverage and composition. Nat. Methods 11, 1144-1146.

Altschup, S.F., Warren, G., Miller, W., Myers, E. W., and Lipman, D. (1990). Basic local alignment search tool. J. Mol. Biol. 215, 403-410.

Apprill, A., Mcnally, S., Parsons, R., and Weber, L. (2015). Minor revision to V4 region SSU rRNA 806R gene primer greatly increases detection of SAR11 bacterioplankton. Aquat. Microb. Ecol. 75, 129-137.

Bengtsson-Palme, J., Hartmann, M., Eriksson, K.M., Pal, C., Thorell, K., Larsson, D.G.J., and Nilsson, R.H. (2015). METAXA2: improved identification and taxonomic classification of small 
and large subunit rRNA in metagenomic data. Mol. Ecol. Resour. 15, 1403-1414.

Bernt, M., Donath, A., Jühling, F., Externbrink, F., Florentz, C., Fritzsch, G., Pütz, J., Middendorf, M., and Stadler, P.F. (2013). MITOS: improved de novo metazoan mitochondrial genome annotation. Mol. Phylogenet. Evol. 69, 313-319.

Bignell, D.E., and Eggleton, P. (1995). On the elevated intestinal pH of higher termites (Isoptera: Termitidae). Ins. Soc. 42, 57-69.

Bignell, D., Eggleton, P., Nunes, L., and Thomas, K. (1997). Termites as mediators of forest carbon fluxes in tropical forests: budgets for carbon dioxide and methane emissions. In Forests and insects, Watt, A.D., Stork, N.E., and Hunter, M.D., ed. (Chapman and Hall, London), pp. 109134.

Bolger, A.M., Lohse, M., and Usadel, B. (2014). Trimmomatic: a flexible trimmer for Illumina sequence data. Bioinformatics 30, 2114-2120.

Bourguignon, T., Šobotník, J., Lepoint, G., Martin, J.M., Hardy, O.J., Dejean, A., and Roisin, Y. (2011). Feeding ecology and phylogenetic structure of a complex neotropical termite assemblage, revealed by nitrogen stable isotope ratios. Ecol. Entomol. 36, 261-269.

Bourguignon, T., Lo, N., Cameron, S.L., Šobotník, J., Hayashi, Y., Shigenobu, S., Watanabe, D., Roisin, Y., Miura, T., and Evans, T.A. (2015). The evolutionary history of termites as inferred from 66 mitochondrial genomes. Mol. Biol. Evol. 32, 406-421.

Bourguignon, T., Lo, N., Šobotník, J., Sillam-Dussès, D., Roisin, Y., and Evans, T.A. (2016). Oceanic dispersal, vicariance and human introduction shaped the modern distribution of the termites Reticulitermes, Heterotermes and Coptotermes. Proc. R. Soc. B. 283, 20160179.

Bourguignon, T., Lo, N., Sobotnik, J., Ho, S.Y.W., Iqbal, N., Coissac, E., Lee, M., Jendryka, M.M., Sillam-Dussès, D., Krizkova, B., et al. (2017). Mitochondrial phylogenomics resolves the global spread of higher termites, ecosystem engineers of the tropics. Mol. Biol. Evol. 34, 589-597.

Bourguignon, T., Lo, N., Dietrich, C., Šobotník, J., Sidek, S., Roisin, Y., Brune, A., and Evans, T.A. (2018). Rampant host switching shaped the termite gut microbiome. Curr. Biol. 28, 649-654.

Bowers, R.M., Kyrpides, N.C., Stepanauskas, R., Harmon-Smith, M., Doud, D., Reddy, T.B.K., Schulz, F., Jarett, J., Rivers, A.R., Eloe-Fadrosh, E.A., et al. (2017). Minimum information about a single amplified genome (MISAG) and a metagenome-assembled genome (MIMAG) of bacteria and archaea. Nat. Biotechnol. 35, 725-731.

Brauman, A., Koenig, J.F., Dutreix, J., and Garcia, J.L. (1990). Characterization of two sulfatereducing bacteria from the gut of the soil-feeding termite, Cubitermes speciosus. Antonie van Leeuwenhoek 58, 271-275.

Brauman, A., Kane, M.D., Labat, M., and Breznak, J.A. (1992). Genesis of acetate and methane by gut bacteria of nutritionally diverse termites. Science $257,1384-1387$.

Breznak, J.A., and Switzer, J.M. (1986). Acetate synthesis from $\mathrm{H}_{2}$ plus $\mathrm{CO}_{2}$ by termite gut microbes. Appl. Environ. Microbiol. 52, 623-630.

Breznak, J.A. (2000). Ecology of prokaryotic microbes in the guts of wood and litter-feeding termites. In Termites: evolution, sociality, symbioses, ecology, Abe, T., Bignell, D. E., and Higashi, M., ed. (Kluwer Academic Publishers), pp. 209-231. 
Brune, A., and Ohkuma, M. (2011). Role of the termite gut microbiota in symbiotic digestion. In Biology of termites: A modern synthesis, Bignell, D.E., Roisin, Y., and Lo, N., ed. (Springer), pp. 439-475.

Brune, A. (2014). Symbiotic digestion of lignocellulose in termite guts. Nat. Rev. Microbiol. 12, $168-180$.

Brune, A. (2018). Methanogens in the digestive tract of termites. In (Endo)symbiotic methanogenic archaea. Hackstein, J.H.P, ed. (Book series: Microbiology monographs vol-19. 2nd ed. Springer, Cham), pp. 81-101.

Brune, A. (2019). Methanogenesis in the digestive tracts of insects and other arthropods. In Biogenesis of hydrocarbons, Stams, A.J.M., Sousa, D.E., ed. (Book series: Handbook of hydrocarbon and lipid microbiology. Springer, Cham), pp. 229-260.

Bucek, A., Šobotník, J., He, S., Shi, M., McMahon, D.P., Holmes, E.C., Roisin, Y., Lo, N., and Bourguignon, T. (2019). Evolution of termite symbiosis informed by transcriptome-based phylogenies. Curr. Biol. 29, 3728-3734.

Buchfink, B., Xie, C., and Huson, D.H. (2015). Fast and sensitive protein alignment using DIAMOND. Nat. Methods 12, 59-60.

Buckel, W., and Thauer, R.K. (2013). Energy conservation via electron bifurcating ferredoxin reduction and proton/Na+ translocating ferredoxin oxidation. Biochimica et Biophysica Acta 1827, 94-113.

Calusinska, M., Marynowska, M., Bertucci, M., Untereiner, B., Klimek, D., Goux, X., SillamDussès, D., Gawron, P., Halder, R., Wilmes, P., et al. (2020). Integrative omics analysis of the termite gut system adaptation to Miscanthus diet identifies lignocellulose degradation enzymes. Commun. Biol. 3, 275.

Chan, P.P. and Lowe, T.M. (2019). tRNAscan-SE: Searching for tRNA genes in genomic sequences. Methods Mol. Biol. 1962, 1-14.

Chaumeil, P.A., Mussig, A.J., Hugenholtz, P., and Parks, D.H. (2020). GTDB-Tk: a toolkit to classify genomes with the Genome Taxonomy Database. Bioinformatics 36, 1925-1927.

Desai, M.S., and Brune, A. (2012). Bacteroidales ectosymbionts of gut flagellates shape the nitrogen-fixing community in dry-wood termites. ISME J. 6, 1302-1313.

Dietrich, C., Köhler, T., and Brune, A. (2014). The cockroach origin of the termite gut microbiota: Patterns in bacterial community structure reflect major evolutionary events. Appl. Environ. Microbiol. 80, 2261-2269.

Donovan, S.E., Eggleton, P., and Bignell, D.E. (2001). Gut content analysis and a new feeding group classification of termites. Ecol. Entomol. 26, 356-366.

Dos Santos, P.C., Fang, Z., Mason, S.W., Setubal, J.C., and Dixon, R. (2012). Distribution of nitrogen fixation and nitrogenase-like sequences amongst microbial genomes. BMC Genomics 13, 162.

Dröge, S., Limper, U., Emtiazi, F., Schönig, I., Pavlus, N., Drzyzga, O., Fischer, U., and König, H. (2005). In vitro and in vivo sulfate reduction in the gut contents of the termite Mastotermes darwiniensis and the rose-chafer Pachnoda marginata. J. Gen. Appl. Microbiol. 51, 57-64. 
Drummond, A.J., Ho, S.Y.W., Phillips, M.J., and Rambaut, A. (2006). Relaxed phylogenetics and dating with confidence. PLoS Biol. 4, e88.

Edgar, R.C. (2018). Accuracy of taxonomy prediction for 16S rRNA and fungal ITS sequences. PeerJ 6, e4652.

El-Gebali, S., Mistry, J., Bateman, A., Eddy, S.R., Potter, S.C., Qureshi, M., Richardson, L.J., Salazar, G.A., Smart, A., Sonnhammer, E.L.L., et al. (2019). The Pfam protein families database in 2019. Nucleic Acids Res. 47, 427-432.

Evans, P.N., Boyd, J.A., Leu, A.O., Woodcroft, B.J., Parks, D.H., Hugenholtz, P., and Tyson, G.W. (2019). An evolving view of methane metabolism in the Archaea. Nat. Rev. Microbiol. 17, 219232.

Gernhard, T. (2008). The conditioned reconstructed process. J. Theor. Biol. 253, 769-778.

Gloor, G.B., Macklaim, J.M., Pawlowsky-Glahn, V., and Egozcue, J.J. (2017). Microbiome datasets are compositional: And this is not optional. Front. Microbiol. 8, 2224.

Graber, J.R., and Breznak, J.A. (2004). Physiology and nutrition of Treponema primitia, an H2/CO2-acetogenic spirochete from termite hindguts. Appl. Environ. Microbiol. 70, 1307-1314.

Graham, E.D., Heidelberg, J.F., and Tully, B.J. (2018). Potential for primary productivity in a globally-distributed bacterial phototroph. ISME J. 12, 1861-1866.

Gurevich, A., Saveliev, V., Vyahhi, N., and Tesler, G. (2013). QUAST: quality assessment tool for genome assemblies. Bioinformatics 29, 1072-1075.

He, S., Ivanova, N., Kirton, E., Allgaier, M., Bergin, C., Scheffrahn, R.H., Kyrpides, N.C., Warnecke, F., Tringe, S.G., and Hugenholtz, P. (2013). Comparative metagenomic and metatranscriptomic analysis of hindgut paunch microbiota in wood- and dung-feeding higher termites. PLoS ONE 8, 560-565.

Hervé, V., Liu, P., Dietrich, C., Sillam-Dussès, D., Stiblik, P., Šobotník, J., and Brune, A. (2020). Phylogenomic analysis of 589 metagenome-assembled genomes encompassing all major prokaryotic lineages from the gut of higher termites. PeerJ 8, e8614.

Hongoh, Y., Sharma, V.K., Prakash, T., Noda, S., Toh, H., Taylor, T.D., Kudo, T., Sakaki, Y., Toyoda, A., Hattori, M., et al. (2008). Genome of an endosymbiont coupling $\mathrm{N}_{2}$ fixation to cellulolysis within protist cells in termite gut. Science 322, 1108-1109.

Hongoh, Y., and Ohkuma, M. (2010). Termite gut flagellates and their methanogenic and eubacterial symbionts. In (Endo)sumbiotic methanogenic archaea, Hackstein, J.H., ed. (SpringerVerlag), pp. 55-79.

Hongoh, Y. (2011). Toward the functional analysis of uncultivable, symbiotic microorganisms in the termite gut. Cell. Mol. Life Sci. 68, 1311-1325.

Huerta-Cepas, J., Szklarczyk, D., Forslund, K., Cook, H., Heller, D., Walter, M.C., Rattei, T., Mende, D.R., Sunagawa, S., Kuhn, M., et al. (2016). eggNOG 4.5: a hierarchical orthology framework with improved functional annotations for eukaryotic, prokaryotic and viral sequences. Nucleic Acids Res. 44, 286-293.

Hungate, R.E. (1939). Experiments on the nutrition of Zootermopsis. III. The anaerobic carbohydrate dissimilation by the intestinal protozoa. Ecology 20, 230-245. 
Ikeda-Ohtsubo, W., Strassert, J.F.H., Köhler, T., Mikaelyan, A., Gregor, I., McHardy, A.C., Tringe, S.G., Hugenholtz, P., Radek, R., and Brune, A. (2016). 'Candidatus Adiutrix intracellularis', an endosymbiont of termite gut flagellates, is the first representative of a deep-branching clade of Deltaproteobacteria and a putative homoacetogen. Environ. Microbiol. 18, 2548-2564.

Inoue, T., Kitade, O., Yoshimura, T., and Yamaoka, I. (2000). Symbiotic associations with protists. In Termites: evolution, sociality, symbioses, ecology, Abe, T., Bignell, D.E., and Higashi, M., ed. (Kluwer, Dordrechit), pp. 275-288.

Inoue, J.I., Oshima, K., Suda, W., Sakamoto, M., Ino, T., Noda, S., Hongoh, Y., Hattori, M., and Ohkuma, M. (2015). Distribution and evolution of nitrogen fixation genes in the phylum Bacteroidetes. Microbes and Environ. 30, 44-50.

Iwadate, Y., and Kato, J.I. (2019). Identification of a formate-dependent uric acid degradation pathway in Escherichia coli. J. Bacteriol. 201, e00573-18.

Ji, R., and Brune, A. (2001). Transformation and mineralization of ${ }^{14} \mathrm{C}$-labeled cellulose, peptidoglycan, and protein by the soil-feeding termite Cubitermes Orthognathus. Biol. Fertil. Soils $33,166-174$.

Ji, R., and Brune, A. (2005). Digestion of peptidic residues in humic substances by an alkali-stable and humic-acid-tolerant proteolytic activity in the gut of soil-feeding termites. Soil Biol. Biochem. $37,1648-1655$.

Kanehisa, M., Sato, Y., Kawashima, M., Furumichi, M., and Tanabe, M. (2016). KEGG as a reference resource for gene and protein annotation. Nucleic Acids Res. 44, 457-462.

Katoh, K., Misawa, K., Kuma, K., and Miyata, T. (2002). MAFFT: a novel method for rapid multiple sequence alignment based on fast Fourier transform. Nucleic Acids Res. 30, 3059-3066.

Keck, F., Rimet, F., Bouchez, A., and Franc, A. (2016). Phylosignal: An R package to measure, test, and explore the phylogenetic signal. Ecol. Evol. 6, 2774-2780.

Kozich, J. J., Westcott, S. L., Baxter, N. T., Highlander, S. K., and Schloss, P.D. (2013). Development of a dual-index sequencing strategy and curation pipeline for analyzing amplicon sequence data on the MiSeq Illumina sequencing platform. Appl. Environ. Microbiol. 79, 51125120 .

Kuhnigk, T., Branke, J., Krekeler, D., Cypionka, H., and König, H. (1996). A feasible role of sulfate-reducing bacteria in the termite gut. Syst. Appl. Microbiol. 19, 139-149.

Kuwahara, H., Yuki, M., Izawa, K., Ohkuma, M., and Hongoh, Y. (2017). Genome of 'Ca. Desulfovibrio trichonymphae', an $\mathrm{H}_{2}$-oxidizing bacterium in a tripartite symbiotic system within a protist cell in the termite gut. ISME J. 11, 766-776.

Lang, K., Schuldes, J., Klingl, A., Poehlein, A., Daniel, R., and Brune, A. (2015). New mode of energy metabolism in the seventh order of methanogens as revealed by comparative genome analysis of "Candidatus Methanoplasma termitum." Appl. Environ. Microbiol. 81, 1338-1352.

Langmead, B., and Salzberg, S.L. (2013). Fast gapped-read alignment with Bowtie2. Nat. Methods 9, 357-359.

Larkin, M.A., Blackshields, G., Brown, N.P., Chenna, R., Mcgettigan, P.A., McWilliam, H., Valentin, F., Wallace, I.M., Wilm, A., Lopez, R., et al. (2007). Clustal W and Clustal X version 


\subsection{Bioinformatics 23, 2947-2948.}

Leadbetter, J.. R., Schmidt, T.. M., Graber, J.. R., and Breznak, J.A. (1999). Acetogenesis from $\mathrm{H}_{2}$ plus $\mathrm{CO}_{2}$ by spirochetes from termite guts. Science $283,686-689$.

Lilburn, K., Ostrom, N., Byzek, K., Leadbetter, J., Breznak, J., and Kim, T. (2001). Nitrogen fixation by symbiotic and free living spirochetes. Science 292, 2495-2498.

Liu, C., Zou, G., Yan, X., and Zhou, X. (2018). Screening of multimeric $\beta$-xylosidases from the gut microbiome of a higher termite, Globitermes brachycerastes. Int. J. Biol. Sci. 14, 608-615.

Lo, N., Tokuda, G., Watanabe, H., Rose, H., Slaytor, M., Maekawa, K., Bandi, C., and Noda, H. (2000). Evidence from multiple gene sequences indicates that termites evolved from wood-feeding cockroaches. Curr. Biol. 10, 801-804.

Loh, H.Q., Hervé, V., and Brune, A. (2021). Metabolic potential for reductive acetogenesis and a novel energy-converting [NiFe] hydrogenase in Bathyarchaeia from termite guts - A genomecentric analysis. Front. Microbiol. 11, 635786.

Lombard, V., Ramulu, H.G., Drula, E., Coutinho, P.M., and Henrissat, B. (2014). The carbohydrate-active enzymes database (CAZy) in 2013. Nucleic Acids Res. 42, 490-495.

Marynowska, M., Goux, X., Sillam-Dussès, D., Rouland-Lefèvre, C., Halder, R., Wilmes, P., Gawron, P., Roisin, Y., Delfosse, P., and Calusinska, M. (2020). Compositional and functional characterisation of biomass-degrading microbial communities in guts of plant fibre- and soilfeeding higher termites. Microbiome 8, 96.

Mendler, K., Chen, H., Parks, D.H., Lobb, B., Hug, L.A., and Doxey, A.C. (2019). AnnoTree: visualization and exploration of a functionally annotated microbial tree of life. Nucleic Acids Res. $47,4442-4448$.

Michaud, C., Hervé, V., Dupont, S., Dubreuil, G., Bézier, A.M., Meunier, J., Brune, A., and Dedeine, F. (2020). Efficient but occasionally imperfect vertical transmission of gut mutualistic protists in a wood-feeding termite. Mol. Ecol. 29, 308-324.

Mikaelyan, A., Strassert, J.F.H., Tokuda, G., and Brune, A. (2014). The fibre-associated cellulolytic bacterial community in the hindgut of wood-feeding higher termites (Nasutitermes spp.). Environ. Microbiol. 16, 2711-2722.

Mikaelyan, A., Dietrich, C., Köhler, T., Poulsen, M., Sillam-Dussès, D., and Brune, A. (2015). Diet is the primary determinant of bacterial community structure in the guts of higher termites. Mol. Ecol. 24, 5284-5295.

Mikaelyan, A., Thompson, C.L., Meuser, K., Zheng, H., Rani, P., Plarre, R., and Brune, A. (2017). High-resolution phylogenetic analysis of Endomicrobia reveals multiple acquisitions of endosymbiotic lineages by termite gut flagellates. Environ. Microbiol. Rep. 9, 477-483.

Nalepa, C. (2017). What kills the hindgut flagellates of lower termites during the host molting cycle? Microorganisms 5:82.

Nalepa, C.A. (1991). Ancestral transfer of symbionts between cockroaches and termites: an alternative hypothesis. Proc. R. Soc. B 246, 191-195.

Ngugi, D.K., Ji, R., and Brune, A. (2011). Nitrogen mineralization, denitrification, and nitrate ammonification by soil-feeding termites: a ${ }^{15} \mathrm{~N}$-based approach. Biogeochemistry 103, 355-369. 
Ngugi, D.K., and Brune, A. (2012). Nitrate reduction, nitrous oxide formation, and anaerobic ammonia oxidation to nitrite in the gut of soil-feeding termites (Cubitermes and Ophiotermes spp.). Environ. Microbiol. 14, 860-871.

Nishimura, Y., Otagiri, M., Yuki, M., Shimizu, M., Inoue, J., Moriya, S., and Ohkuma, M. (2020). Division of functional roles for termite gut protists revealed by single-cell transcriptomes. ISME J. 14, 2449-2460.

Nurk, S., Meleshko, D., Korobeynikov, A., and Pevzner, P.A. (2017). metaSPAdes: a new versatile metagenomic assembler. Genome Res. 27, 824-834.

Ochman, H., Lawrence, J.G., and Groisman, E.A. (2000). Lateral gene transfer and the nature of bacterial innovation. Nature 405, 299-304.

Ohkuma, M., Noda, S., and Kudo, T. (1999). Phylogenetic diversity of nitrogen fixation genes in the symbiotic microbial community in the gut of diverse termites. Appl. Environ. Microbiol. 65, 4926-4934.

Ohkuma, M., Noda, S., Hongoh, Y., and Kudo, T. (2001). Coevolution of symbiotic systems of termites and their gut microorganisms. Ecology 41, 73-74.

Ohkuma, M., and Brune, A. (2011). Diversity, structure, and evolution of the termite gut microbial community. In Biology of termites: a modern synthesis. Bignell, D.E, Roisin, Y. and Lo, N., ed. (Springer), pp. 413-138.

Ohkuma, M., Noda, S., Hattori, S., Iida, T., Yuki, M., Starns, D., Inoue, J.I., Darby, A.C., and Hongoh, Y. (2015). Acetogenesis from $\mathrm{H}_{2}$ plus $\mathrm{CO}_{2}$ and nitrogen fixation by an endosymbiotic spirochete of a termite-gut cellulolytic protist. Proc. Natl. Acad. Sci. USA 112, 10224-10230.

Papudeshi, B., Haggerty, J.M., Doane, M., Morris, M.M., Walsh, K., Beattie, D.T., Pande, D., Zaeri, P., Silva, G.G.Z., Thompson, F., et al. (2017). Optimizing and evaluating the reconstruction of metagenome-assembled microbial genomes. BMC Genomics 18, 915.

Parada, A.E., Needham, D.M., and Fuhrman, J.A. (2016). Every base matters: Assessing small subunit rRNA primers for marine microbiomes with mock communities, time series and global field samples. Environ. Microbiol. 18, 1403-1414.

Parks, D.H., Imelfort, M., Skennerton, C.T., Hugenholtz, P., and Tyson, G.W. (2015). CheckM: assessing the quality of microbial genomes recovered from. Genome Res. 25, 1043-1055.

Parks, D.H., Chuvochina, M., Chaumeil, P.A., Rinke, C., Mussig, A.J., and Hugenholtz, P. (2020). A complete domain-to-species taxonomy for bacteria and archaea. Nat. Biotechnol. 38, 1079-1086.

Paul, K., Nonoh, J.O., Mikulski, L., and Brune, A. (2012). "Methanoplasmatales," thermoplasmatales-related archaea in termite guts and other environments, are the seventh order of methanogens. Appl. Environ. Microbiol. 78, 8245-8253.

Pester, M., and Brune, A. (2007). Hydrogen is the central free intermediate during lignocellulose degradation by termite gut symbionts. ISME J. 1, 551-565.

Potrikus, C.J., and Breznak, J.A. (1980). Uric acid-degrading bacteria in guts of termites [Reticulitermes flavipes (Kollar)]. Appl. Environ. Microbiol. 40, 117-124.

Potrikus, C.J., and Breznak, J.A. (1981). Gut bacteria recycle uric acid nitrogen in termites: A strategy for nutrient conservation. Proc. Natl. Acad. Sci. USA 78, 4601-4605. 
Poulsen, M., Hu, H., Li, C., Chen, Z., Xu, L., Otani, S., Nygaard, S., Nobre, T., Klaubauf, S., Schindler, P.M., et al. (2014). Complementary symbiont contributions to plant decomposition in a fungus-farming termite. Proc. Natl. Acad. Sci. USA 111, 14500-14505.

Price, M.N., Dehal, P.S., and Arkin, A.P. (2009). FastTree: computing large minimum evolution trees with profiles instead of a distance matrix. Mol. Biol. Evol. 26, 1641-1650.

Quast, C., Pruesse, E., Yilmaz, P., Gerken, J., Schweer, T., Glo, F.O., and Yarza, P. (2013). The SILVA ribosomal RNA gene database project: improved data processing and web-based tools. Nucleic Acids Res. 41, 590-596.

Quinn, T.P., Richardson, M.F., Lovell, D., and Crowley, T.M. (2017). propr: An R-package for identifying proportionally abundant features using compositional data analysis. Sci Rep. 7, 16252.

R Core Team. (2014). R: A language and environment for statistical computing. Available from: http://www.r-project.org/.

Rahman, N.A., Parks, D.H., Willner, D.L., Engelbrektson, A.L., Goffredi, S.K., Warnecke, F., Scheffrahn, R.H., and Hugenholtz, P. (2015). A molecular survey of Australian and North American termite genera indicates that vertical inheritance is the primary force shaping termite gut microbiomes. Microbiome 3, 5.

Rambaut, A., Drummond, A., Xie, D., Baele, G., and Suchard, M. (2018). Posterior summarization in Bayesian phylogenetics using Tracer 1.7. Syst. Biol. 67, 901-904.

Rognes, T., Flouri, T., Nichols, B., Quince, C., and Mahé, F. (2016). VSEARCH: a versatile open source tool for metagenomics. PeerJ 4,e2584.

Rouland-Lefèvre, C. (2000). Symbiosis with fungi. In termites: evolution, sociality, symbioses, ecology, Abe, T., Bignell, D. E., Higashi, T., ed. (Kluwer Academic Publishers), pp. 289-306.

Sato, T., Hongoh, Y., Noda, S., Hattori, S., Ui, S., and Ohkuma, M. (2009). Candidatus Desulfovibrio trichonymphae, a novel intracellular symbiont of the flagellate Trichonympha agilis in termite gut. Environ. Microbiol. 11, 1007-1015.

Schloss, P.D., Westcott, S.L., Ryabin, T., Hall, J.R., Hartmann, M., Hollister, E.B., Lesniewski, R.A., Oakley, B.B., Parks, D.H., Robinson, C.J., et al. (2009). Introducing mothur: Open-source, platform-independent, community-supported software for describing and comparing microbial communities. Appl. Environ. Microbiol. 75, 7537-7541.

Schuchmann, K., and Müller, V. (2012). A bacterial electron-bifurcating hydrogenase. J. Biol. Chem. 287, 31165-31171.

Schuchmann, K., and Müller, V. (2014). Autotrophy at the thermodynamic limit of life: A model for energy conservation in acetogenic bacteria. Nat. Rev. Microbiol. 12, 809-821.

Schuchmann, K., Chowdhury, N.P., and Müller, V. (2018). Complex multimeric [FeFe] hydrogenases: Biochemistry, physiology and new opportunities for the hydrogen economy. Front. Microbiol. 9, 2911.

Seemann, T. (2014). Prokka: rapid prokaryotic genome annotation. Bioinformatics 30, 2068-2069.

Shinzato, N., Matsumoto, T., Yamaoka, I., Oshima, T., and Yamagishi, A. (2001). Methanogenic symbionts and the locality of their host lower termites. Microbes and Environ. 16, 43-47.

Søndergaard, D., Pedersen, C.N.S., and Greening, C. (2016). HydDB: A web tool for hydrogenase 
classification and analysis. Sci. Rep. 6, 34212.

Song, Y., Hervé, V., Radek, R., Pfeiffer, F., Zheng, H., and Brune, A. (2021). Characterization and phylogenomic analysis of Breznakiella homolactica gen. nov. sp. nov. indicate that termite gut treponemes evolved from non-acetogenic spirochetes in cockroaches. Environ. Microbiol. in press.

Srivastava, A., Malik, L., Sarkar, H., Zakeri, M., Almodaresi, F., Soneson, C., Love, M.I., Kingsford, C., and Patro, R. (2020). Alignment and mapping methodology influence transcript abundance estimation. Genome Biol. 21, 239.

Stingl, U., Radek, R., Yang, H., and Brune, A. (2005). "Endomicrobia": Cytoplasmic symbionts of termite gut protozoa form a separate phylum of prokaryotes. Appl. Environ. Microbiol. 71, $1473-1479$.

Suchard, M.A., Lemey, P., Baele, G., Ayres, D.L., Drummond, A.J., and Rambaut, A. (2018). Bayesian phylogenetic and phylodynamic data integration using BEAST 1.10. Virus Evol. 4, vey016.

Sugimoto, A., Inoue, T., Tayasu, I., Miller, L., Takeichi, S., and Abe, T. (1998). Methane and hydrogen production in a termite-symbiont system. Ecol. Res. 13, 241-257.

Sunagawa, S., Mende, D.R., Zeller, G., Izquierdo-Carrasco, F., Berger, S.A., Kultima, J.R., Coelho, L.P., Arumugam, M., Tap, J., Nielsen, H.B., et al. (2013). Metagenomic species profiling using universal phylogenetic marker genes. Nat. Methods 10, 1196-1199.

Tai, V., James, E.R., Nalep, C.A., Scheffrahn, R.H., Perlman, S.J., and Keeling, P.J. (2015). The role of host phylogeny varies in shaping microbial diversity in the hindguts of lower termites. Appl. Environ. Microbiol. 81, 1059-1070.

Tai, V., Carpenter, K.J., Weber, P.K., Nalepa, C.A., Perlman, S.J., and Keeling, P.J. (2016). Genome evolution and nitrogen fixation in bacterial ectosymbionts of a protist inhabiting woodfeeding cockroaches. Appl. Environ. Microbiol. 82, 4682-4695.

Tartar, A., Wheeler, M.M., Zhou, X., Coy, M.R., Boucias, D.G., and Scharf, M.E. (2009). Parallel metatranscriptome analyses of host and symbiont gene expression in the gut of the termite Reticulitermes flavipes. Biotechnol. Biofuels 2, 25.

Terrapon, N., Lombard, V., Gilbert, H.J., and Henrissat, B. (2015). Automatic prediction of polysaccharide utilization loci in Bacteroidetes species. Bioinformatics 31, 647-655.

Thauer, R.K., Kaster, A.K., Seedorf, H., Buckel, W., and Hedderich, R. (2008). Methanogenic archaea: ecologically relevant differences in energy conservation. Nat. Rev. Microbiol. 6, 579591.

Tholen, A., and Brune, A. (1999). Localization and in situ activities of homoacetogenic bacteria in the highly compartmentalized hindgut of soil-feeding higher termites (Cubitermes spp.). Appl. Environ. Microbiol. 65, 4497-4505.

Thong-On, A., Suzuki, K., Noda, S., Inoue, J.I., Kajiwara, S., and Ohkuma, M. (2012). Isolation and characterization of anaerobic bacteria for symbiotic recycling of uric acid nitrogen in the gut of various termites. Microbes Environ. 27, 186-192.

Tokuda, G., Lo, N., Watanabe, H., Arakawa, G., Matsumoto, T., and Noda, H. (2004). Major 
alteration of the expression site of endogenous cellulases in members of an apical termite lineage. Mol. Ecol. 13, 3219-3228.

Tokuda, G., Mikaelyan, A., Fukui, C., Matsuura, Y., Watanabe, H., Fujishima, M., and Brune, A. (2018). Fiber-associated spirochetes are major agents of hemicellulose degradation in the hindgut of wood-feeding higher termites. Proc. Natl. Acad. Sci. USA 115, 11996-12004.

Treitli, S.C., Kolisko, M., Husník, F., Keeling, P.J., and Hampl, V. (2019). Revealing the metabolic capacity of Streblomastix strix and its bacterial symbionts using single-cell metagenomics. Proc. Natl. Acad. Sci. USA 116, 19675-19684.

Uritskiy, G.V, DiRuggiero, J., and Taylor, J. (2018). MetaWRAP - a flexible pipeline for genomeresolved metagenomic data analysis. Microbiome 6, 158.

Větrovský, T., and Baldrian, P. (2013). The variability of the $16 \mathrm{~S}$ rRNA gene in bacterial genomes and its consequences for bacterial community analyses. PLoS ONE 8, e57923.

Vu, V.Q. (2011). ggbiplot: A ggplot2 based biplot. R package version 0.55. Available from: http://github.com/vqv/ggbiplot.

Wang, Q., Garrity, G. M., Tiedje, J. M., and Cole, J.R. (2007). Naive Bayesian classifier for rapid assignment of rRNA sequences into the new bacterial taxonomy. Appl. Environ. Microbiol. 73, 5261-5267.

Wang, M., Buček, A., Šobotník, J., Sillam-Dussès, D., Evans, T.A., Roisin, Y., Lo, N., and Bourguignon, T. (2019). Historical biogeography of the termite clade Rhinotermitinae (Blattodea: Isoptera). Mol. Phylogenet. Evol. 132, 100-104.

Warnecke, F., Luginbu, P., Ivanova, N., Ghassemian, M., Richardson, T.H., Stege, J.T., Cayouette, M., Mchardy, A.C., Djordjevic, G., Aboushadi, N., et al. (2007). Metagenomic and functional analysis of hindgut microbiota of a wood-feeding higher termite. Nature 450, 560-565.

Watanabe, H., Noda, H., Tokuda, G., and Lo, N. (1998). A cellulase gene of termite origin. Nature 394, 330-331.

Watanabe, H., and Tokuda, G. (2010). Cellulolytic systems in insects. Annu. Rev. Entomol. 55, 609-632.

Wu, D., Jospin, G., and Eisen, J.A. (2013). Systematic identification of gene families for use as 'markers' for fhylogenetic and phylogeny-driven ecological studies of bacteria and archaea and their major subgroups. PLoS ONE 8, e77033.

$\mathrm{Wu}, \mathrm{H}$. (2018). Characterizing xylan-degrading enzymes from a putative xylan utilization system derived from termite gut metagenome. PhD thesis, INSA de Toulouse, France.

Yamada, A., Inoue, T., Noda, S., Hongoh, Y., and Ohkuma, M. (2007). Evolutionary trend of phylogenetic diversity of nitrogen fixation genes in the gut community of wood-feeding termites. Mol. Ecol. 16, 3768-3777.

Yamin, M. (1981). Cellulose metabolism by the flagellate Trichonympha from a termite is independent of endosymbiotic bacteria. Science 211, 58-59.

Yin, Y., Mao, X., Yang, J., Chen, X., Mao, F., and Xu, Y. (2012). dbCAN: a web resource for automated carbohydrate-active enzyme annotation. Nucleic Acids Res. 40, 445-451.

Yuki, M., Kuwahara, H., Shintani, M., Izawa, K., Sato, T., Starns, D., Hongoh, Y., and Ohkuma, 
M. (2015). Dominant ectosymbiotic bacteria of cellulolytic protists in the termite gut also have the potential to digest lignocellulose. Environ. Microbiol. 17, 4942-4953.

Zhang, H., Yohe, T., Huang, L., Entwistle, S., Wu, P., Yang, Z., Busk, P.K., Xu, Y., and Yin, Y. (2018). dbCAN2: A meta server for automated carbohydrate-active enzyme annotation. Nucleic Acids Res. 46, 95-101.

Zheng, H., Dietrich, C., Thompson, C.L., Meuser, K., and Brune, A. (2015). Population structure of Endomicrobia in single host cells of termite gut flagellates (Trichonympha spp.). Microbes Environ. 30, 92-98. 
bioRxiv preprint doi: https://doi. org/10.1101/2021.1201.470864: this version posted December 3, 2021. The copyright holder for this preprint (which was not certified by peer review) is the author/funder, who has granted bioRxiv a license to display the preprint in perpetuity. It is made available under aCC-BY-NC-ND 4.0 International license.

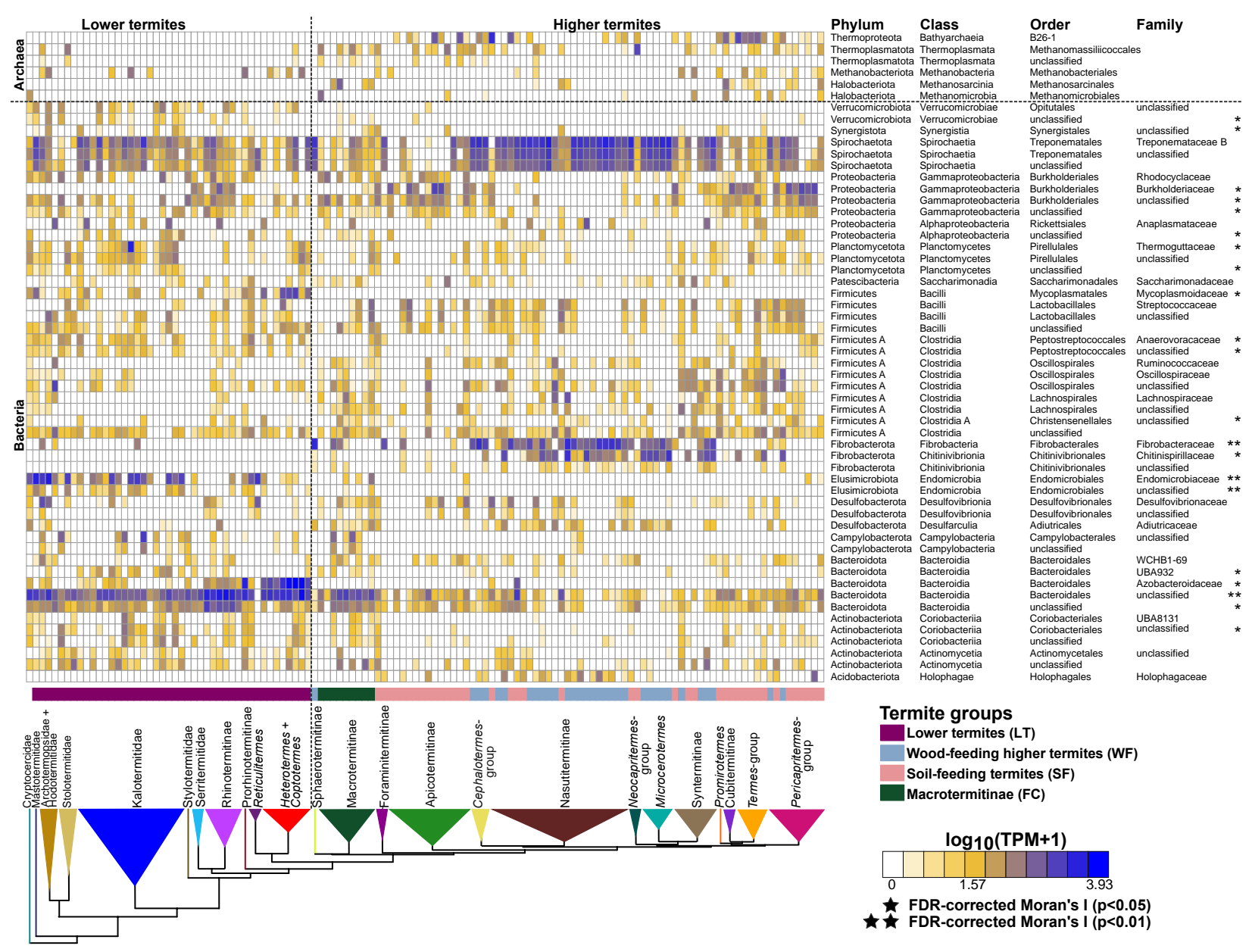

Figure 1. Relative abundance of the top 50 bacterial lineages and the major archaeal orders

found in the gut metagenomes of termites. The relative abundance of prokaryotic taxa was inferred from 40 single-copy marker genes. The color scale represents the logarithm of transcripts per million (TPM). The tree represents a simplified time-calibrated phylogenetic tree reconstructed using host termite mitochondrial genome sequences. Prokaryotic taxa presenting significant phylogenetic autocorrelation with the host phylogeny at a 5\% false discovery rate (FDR) are indicated with an asterisk $(* \mathrm{p}<0.05 ; * * \mathrm{p}<0.01)$. 
bioRxiv preprint doi: https://doi.org/10.1101/2021.12 01.470864 this version posted December 3, 2021. The copyright holder for this preprint (which was not certified by peer review) is the author/funder, who has granted bioRxiv a license to display the preprint in perpetuity. It is made available under aCC-BY-NC-ND 4.0 International license.

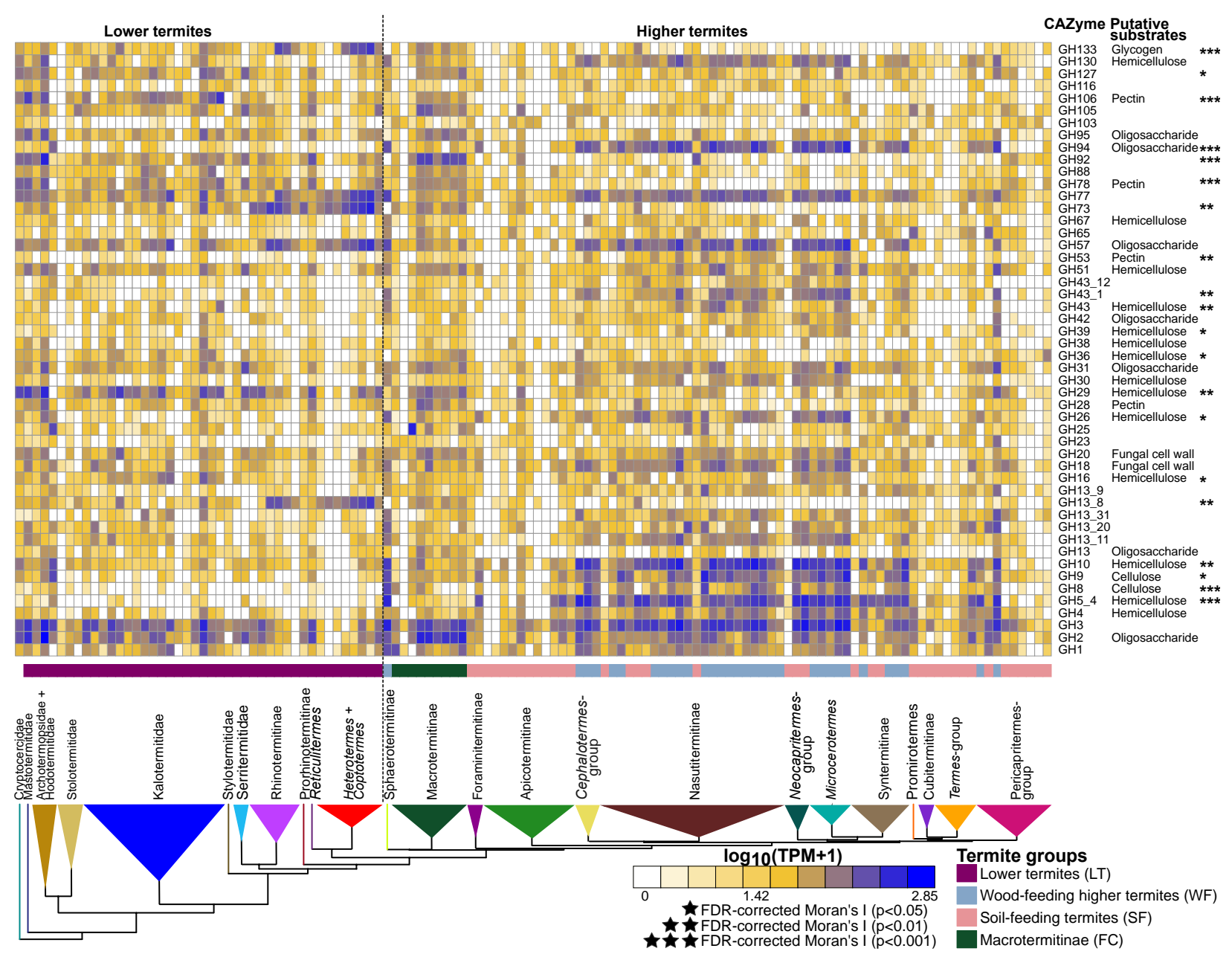

Figure 2. Relative abundance of CAZymes found in gut metagenomes of termites. The heatmap shows the 50 most abundant CAZymes. The color scale represents the logarithm of transcripts per million (TPM). The tree represents a simplified time-calibrated phylogenetic tree reconstructed using host termite mitochondrial genomes. Genes showing significant phylogenetic autocorrelation with the host phylogeny at a 5\% false discovery rate (FDR) are indicated with asterisks $(* \mathrm{p}<0.05 ; * \mathrm{p}<0.01)$. 

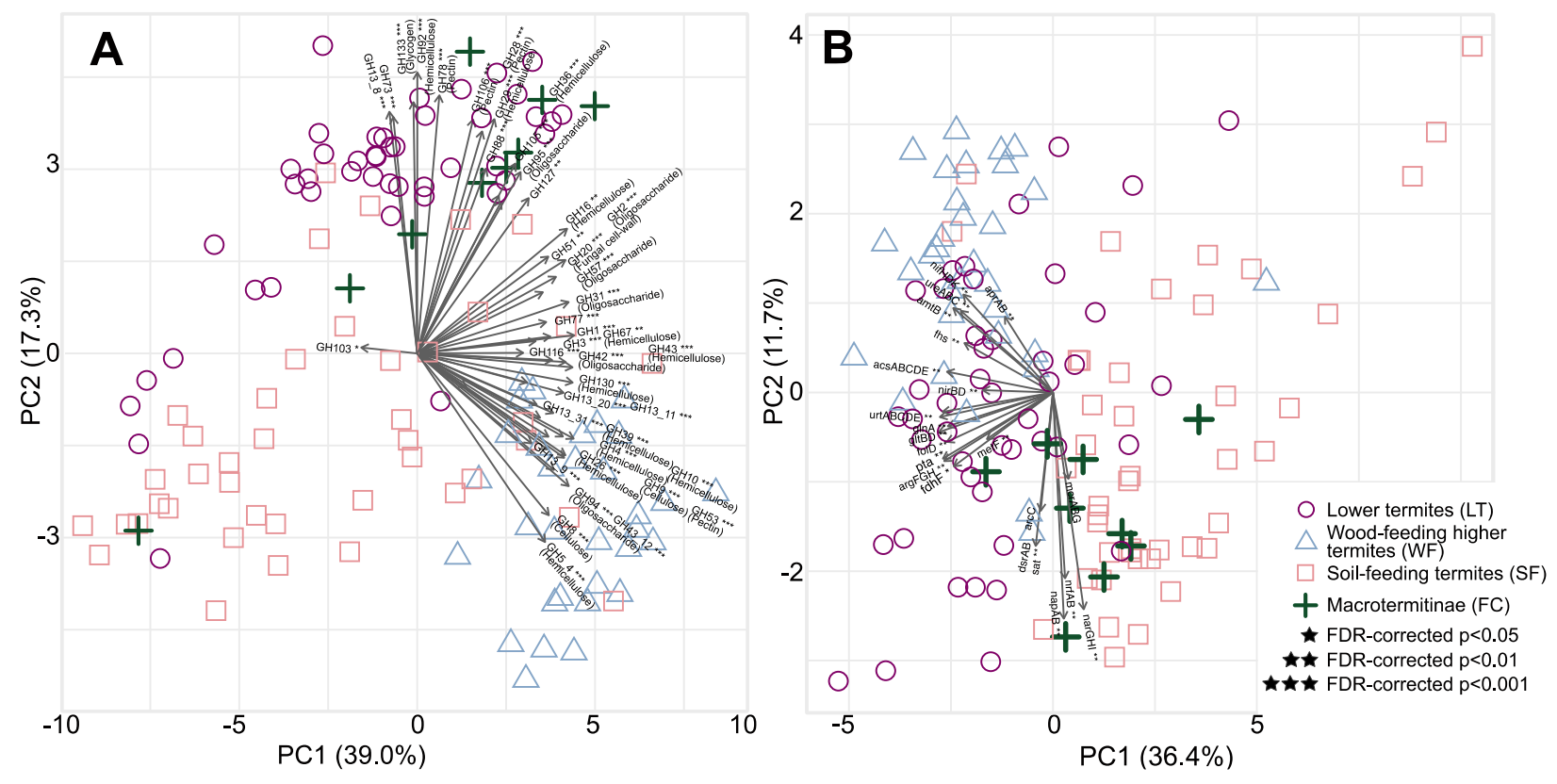

Figure 3. Principle component analysis (PCA) bi-plots showing the distribution of prokaryotic genes involved in lignocellulose digestion in the gut of termites. (A) PCA performed on the relative abundance of 346 CAZymes found in 129 gut metagenome assemblies. The 50 glycoside hydrolases (GHs) that contributed the most to separation of termite diets are plotted (see Table S7). (B) PCA inferred from relative abundance of metabolic genes involved in lignocellulose digestion after carbohydrate degradation. The symbols indicate host feeding habits. The species identity of each data point is available in Table S1. Asterisks indicate significant differences among the four termite groups at $5 \%$ false discovery rate (FDR, $* \mathrm{p}<0.05 ; * * \mathrm{p}<0.01$; $* * * \mathrm{p}<0.001)$ 
bioRxiv preprint doi: https://doi. org/10.1101/2021.12.01.470864 this version posted December 3, 2021. The copyright holder for this preprint (which was not certified by peer review) is the author/funder, who has granted bioRxiv a license to display the preprint in perpetuity. It is made available under aCC-BY-NC-ND 4.0 International license.

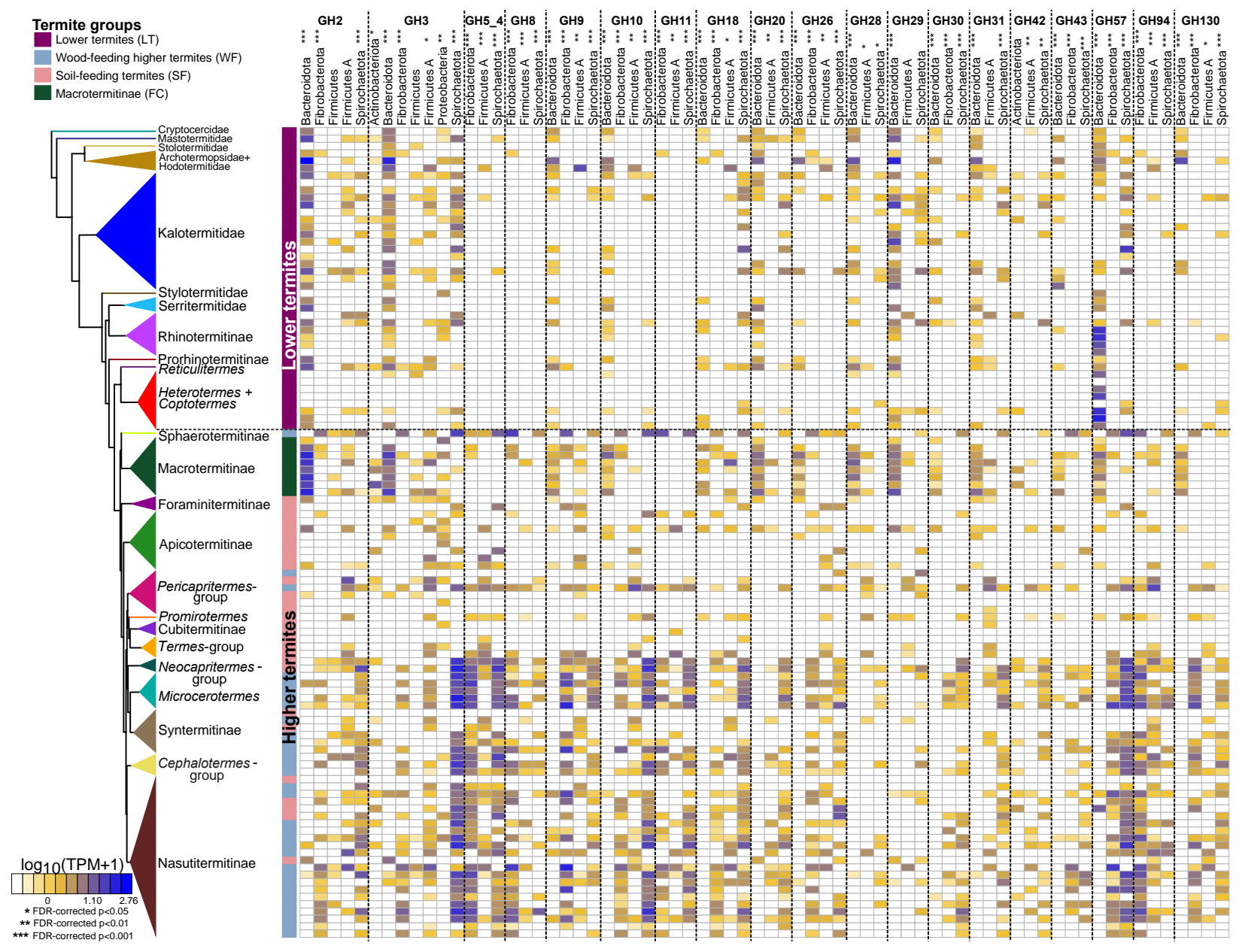

Figure 4. CAZyme families, and their taxonomic origin, for enzymes derived from contigs

longer than 5000 bps and present in $10 \%$ of gut metagenomes. The color scale represents the log-transformed transcripts per million (TPM). The tree represents a simplified time-calibrated phylogenetic tree reconstructed using host termite mitochondrial genomes. Asterisks indicate significant differences among the four termite groups at $5 \%$ false discovery rate (FDR, ${ }^{*} \mathrm{p}<0.05$; $* * \mathrm{p}<0.01 ; * * \mathrm{p}<0.001)$. 
bioRxiv preprint doi: https://doi.org/10.1101/2021.12.01.470864; this version posted December 3, 2021. The copyright holder for this preprint (which was not certified by peer review) is the author/funder, who has granted bioRxiv a license to display the preprint in perpetuity. It is made available under aCC-BY-NC-ND 4.0 International license.
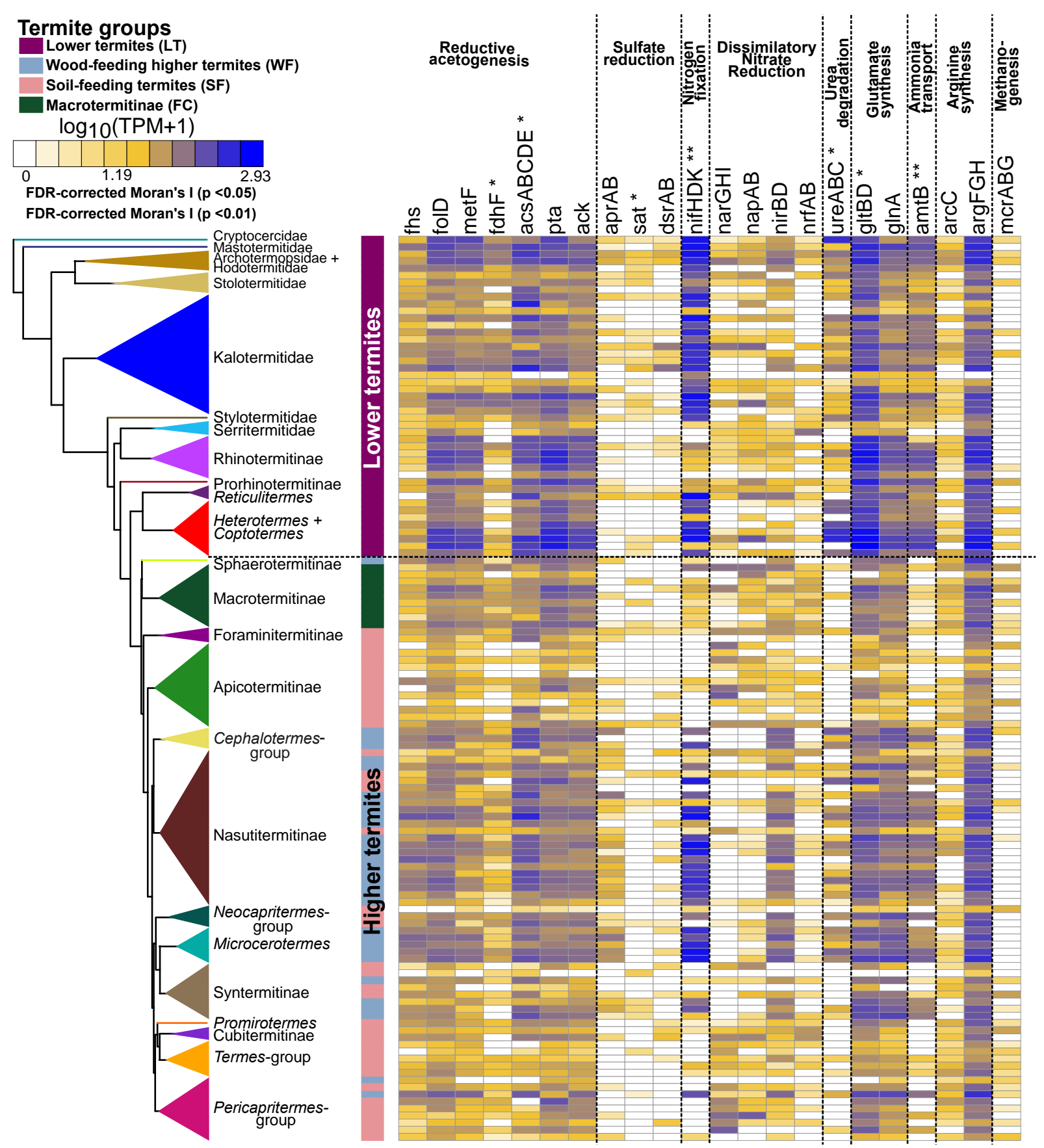

Figure 5. Relative abundance of prokaryotic genes belonging to metabolic pathways involved in the final steps of the lignocellulose digestion in the gut of termites. The color scale represents the logarithm of transcripts per million (TPM). The tree represents a simplified timecalibrated phylogenetic tree reconstructed using host termite mitochondrial genomes. Full names of the gene families and their corresponding KEGG IDs are available in Table S11. 


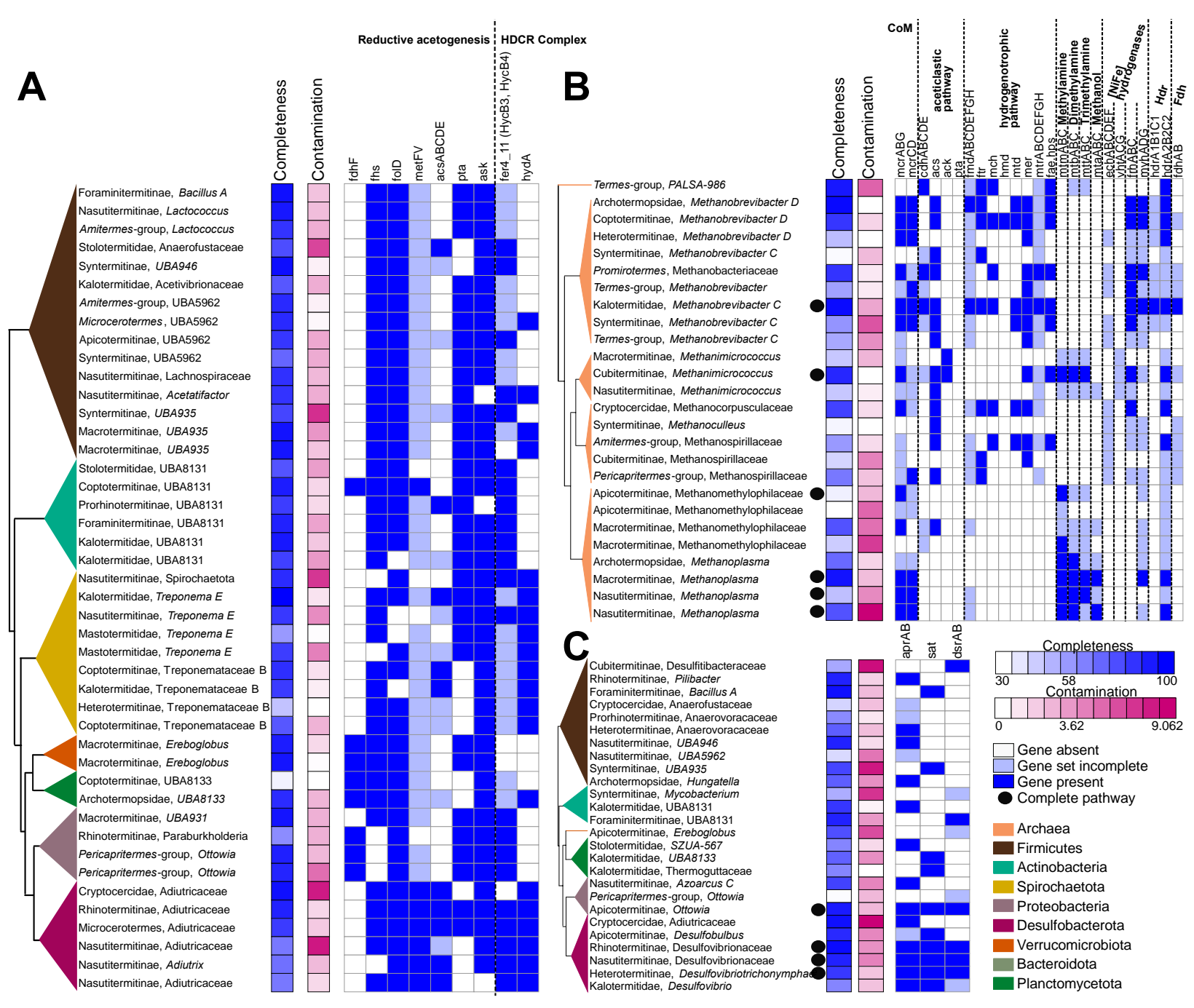

Figure 6. Metabolic pathways involved in the final steps of lignocellulose digestion found in gut metagenome assembled genomes (MAGs) reconstructed in this study. (A) Genes involved in reductive acetogenesis, (B) methanogenesis, and (C) sulfate reduction found in MAGs. The trees represent simplified maximum likelihood phylogenetic trees of the MAGs reconstructed using 43 single-copy marker genes. MAG completeness and contamination, based on CheckM analyses, is shown beside the tree. Dark blue squares indicate gene presence, light blue squares indicate that incomplete gene sets, and open squares indicate gene absence. Detailed information on the gene families and their KEGG IDs are available in Tables S12, S14, and S15. 

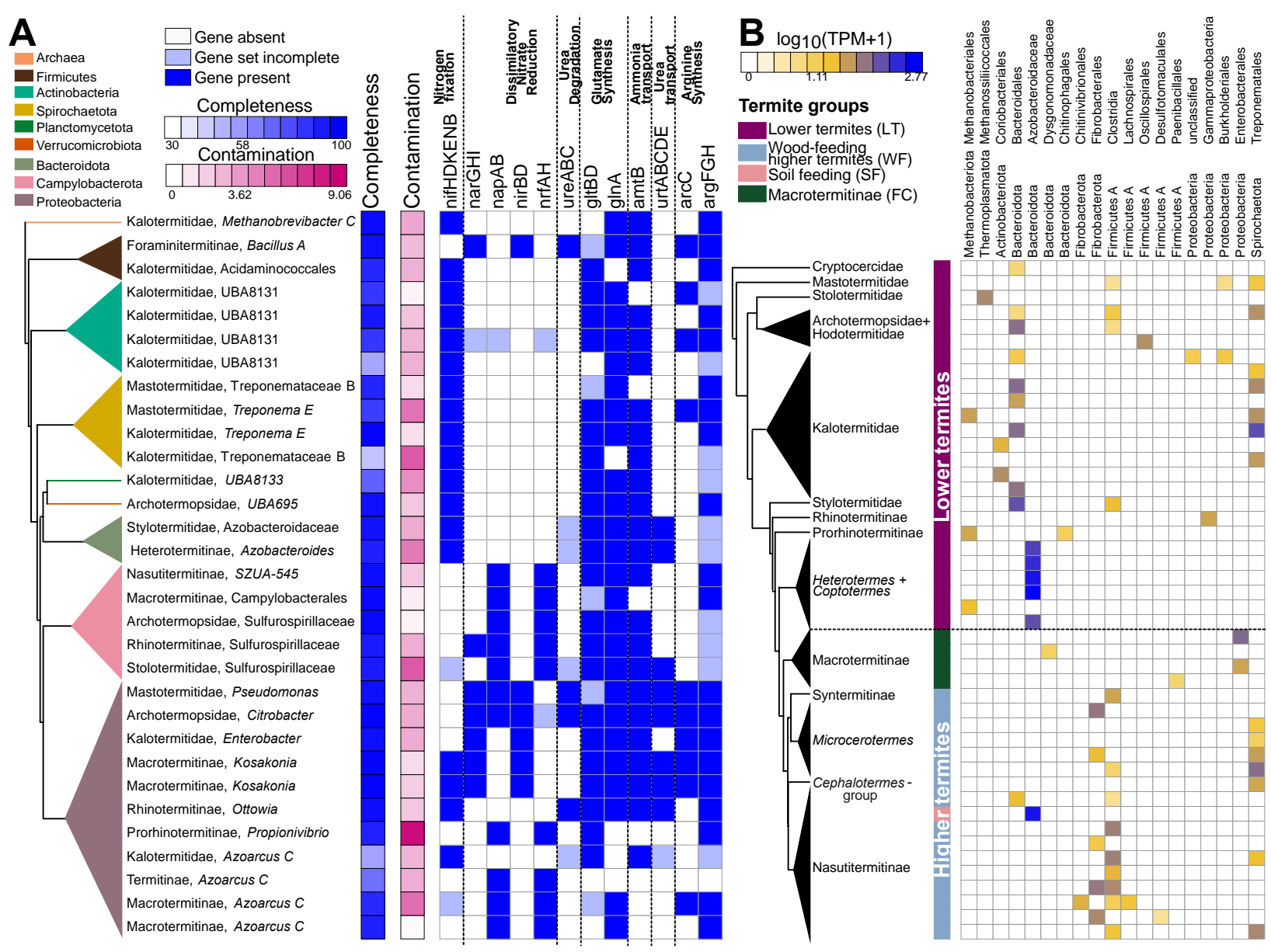

Figure 7. Nitrogen metabolism in the gut of termites. (A) Metagenome-assembled genomes (MAGs) with complete nitrogen fixation or dissimilatory nitrate reduction pathways. All pathways potentially involved in the nitrogen metabolism, namely nitrogen fixation, dissimilatory nitrate reduction, ureases, glutamate metabolism, ammonia transport, urea transport, and arginine metabolism are represented. The tree represents a simplified maximum likelihood phylogenetic tree of the MAGs inferred from 43 marker genes. Completeness and contamination of MAGs, based on CheckM analysis, are shown beside the tree. Dark blue squares indicate gene presence, light blue squares indicate that incomplete gene sets, and open squares indicate gene absence. (B) Abundance of NifHDK operons (nifHDK, vnfHDK, or anfHDK) present in contigs longer than 5000 bps across gut metagenomes. The color scale represents the log-transformed transcripts per million (TPM). The tree represents a simplified time-calibrated phylogenetic tree reconstructed using host termite mitochondrial genomes. 
bioRxiv preprint doi: https://doi.org/10.1101/2021.12.01.470864 this version posted December 3, 2021. The copyright holder for this preprint (which was not certified by peer review) is the author/funder, who has granted bioRxiv a license to display the preprint in perpetuity. It is made available under aCC-BY-NC-ND 4.0 International license.

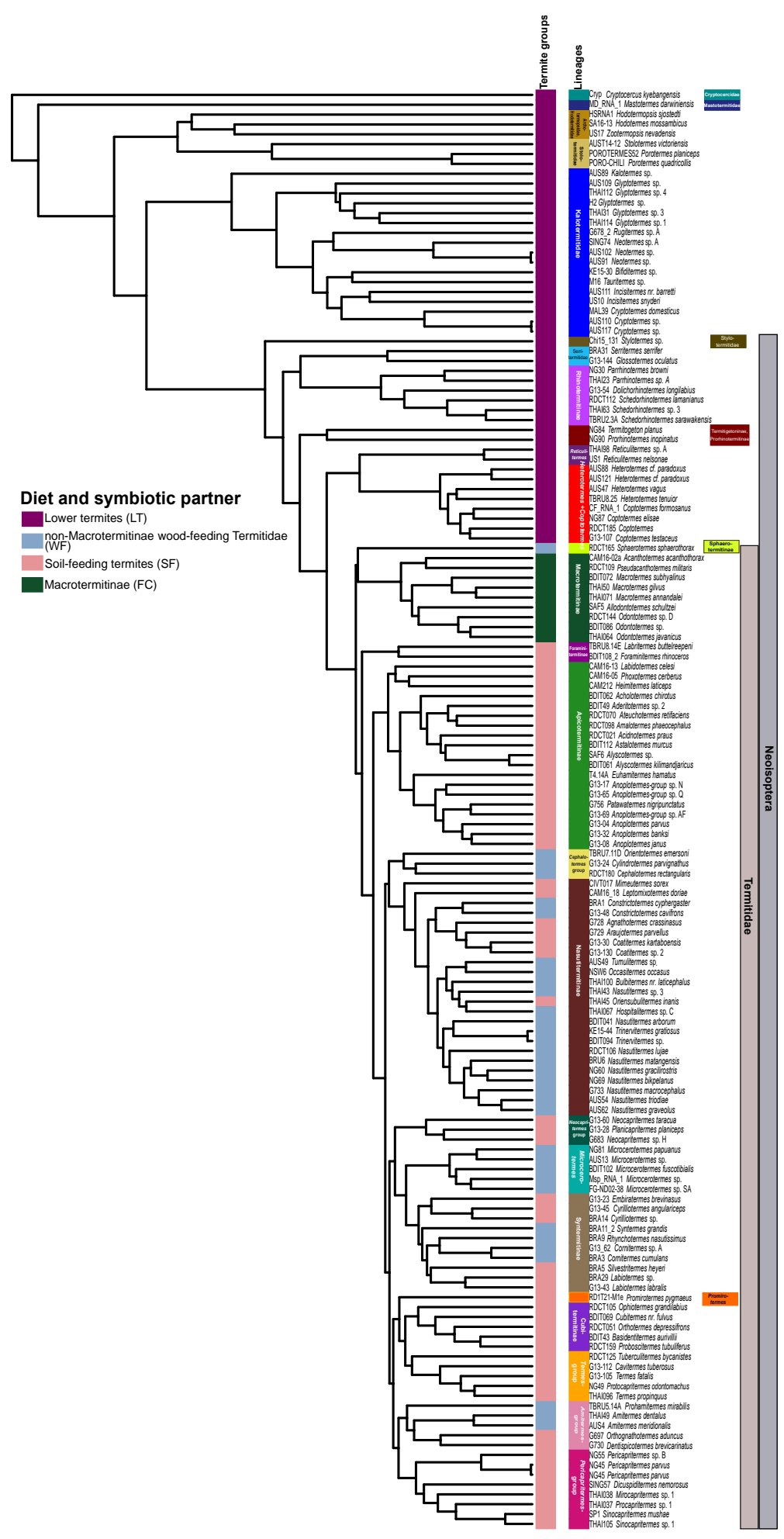

Figure S1. Time-calibrated phylogenetic tree of termites inferred from mitochondrial genome sequences. 
A

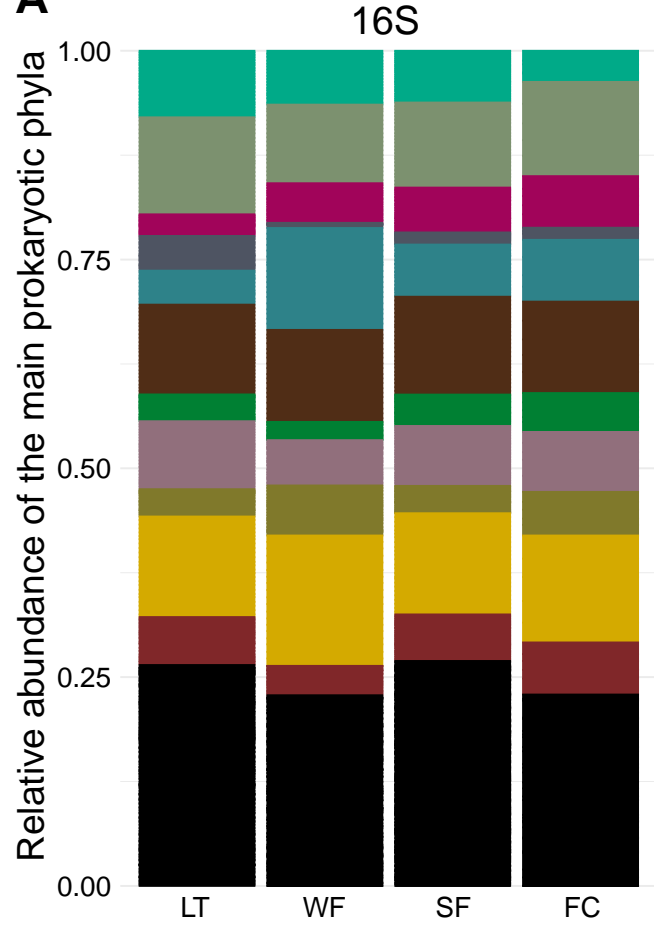

B

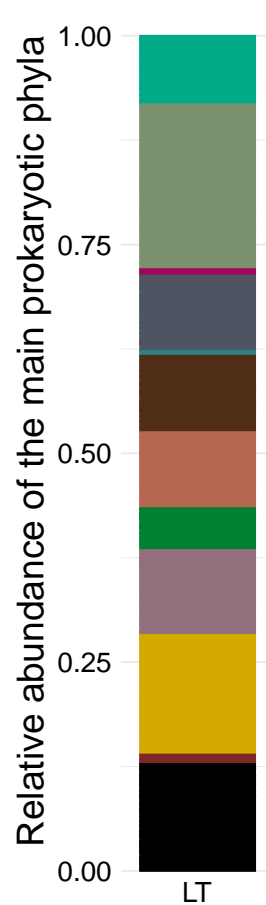

Marker genes

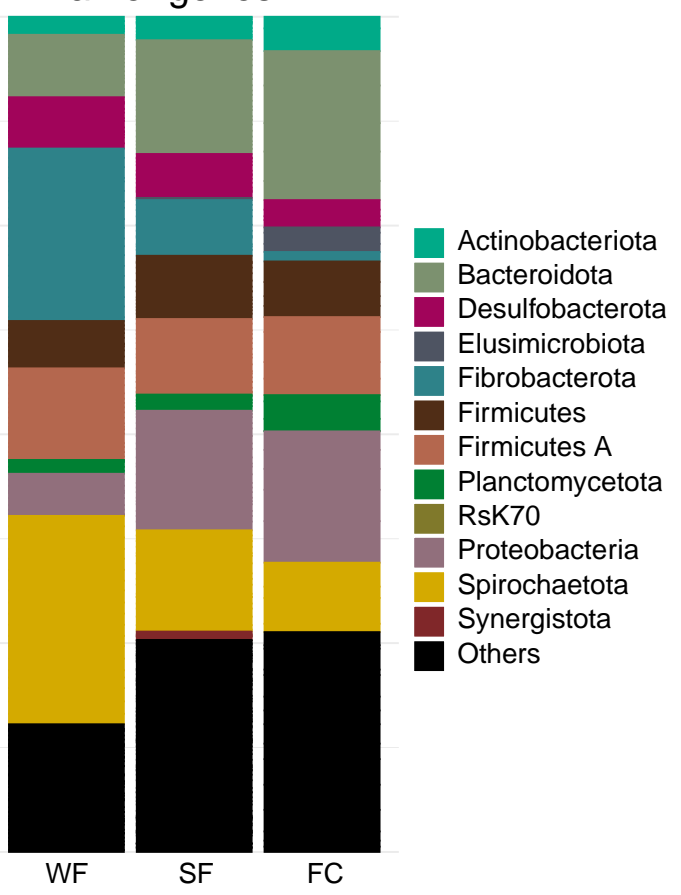

Figure S2. Relative abundance of archaeal and bacterial phyla inferred from the termite gut metagenomes and the 16S rRNA amplicon data of 74 termite samples. 

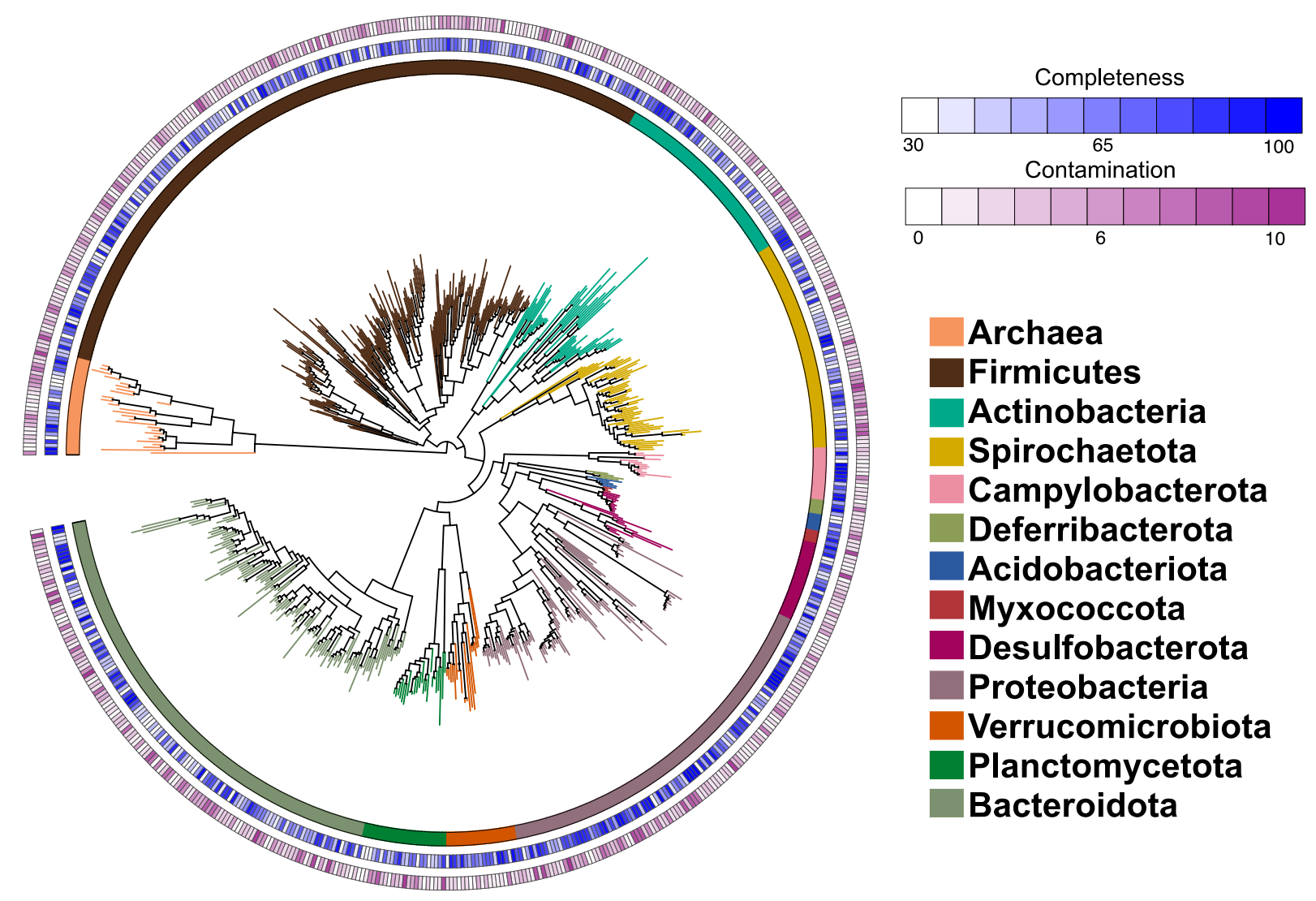

Figure S3. Maximum likelihood phylogenetic tree inferred from 43 single-copy marker genes of 654 metagenome-assembled genomes (MAGs). The completeness and contamination of MAGs was inferred with CheckM (Park et al., 2015). Detailed information about each MAG is available in Table S9. 
bioRxiv preprint doi: https://doi.org/10.1101/2021.12.01.470864; this version posted December 3, 2021. The copyright holder for this preprint (which was not certified by peer review) is the author/funder, who has granted bioRxiv a license to display the preprint in perpetuity. It is made available under aCC-BY-NC-ND 4.0 International license.
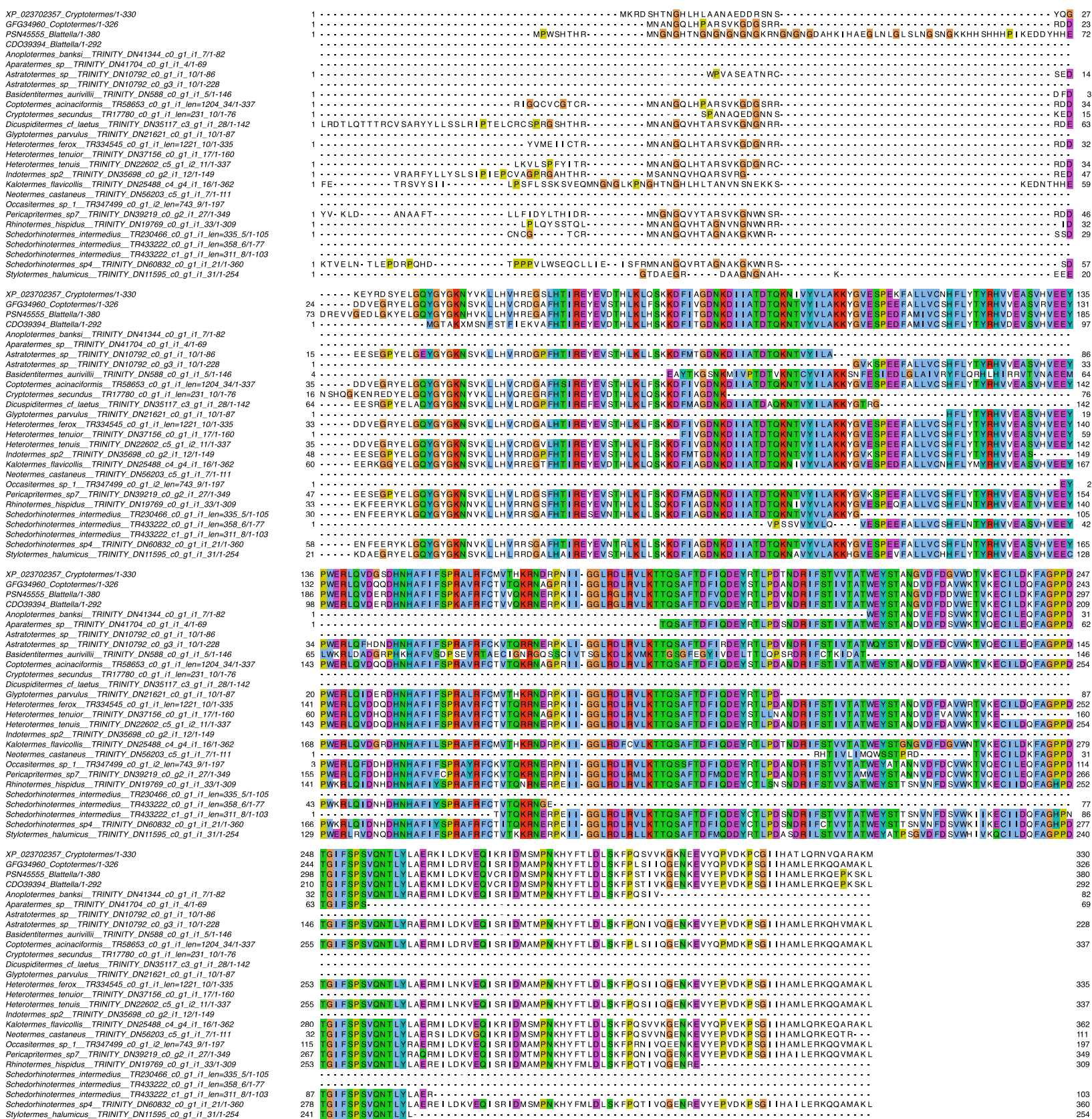

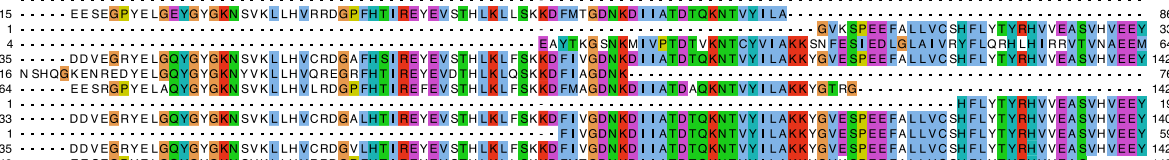

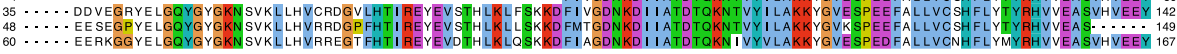

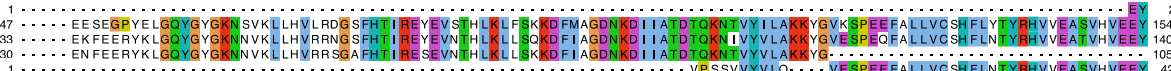

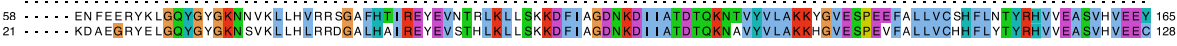

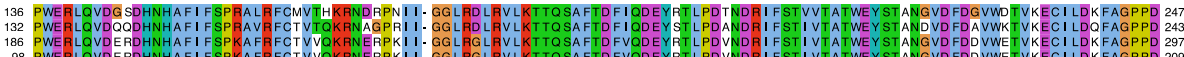

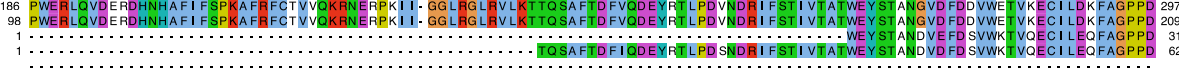

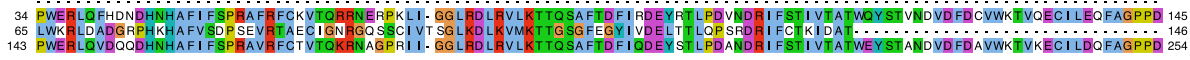

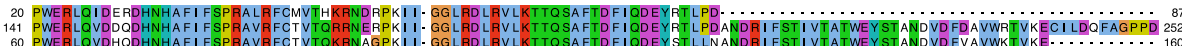

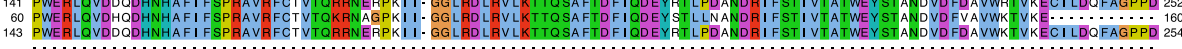

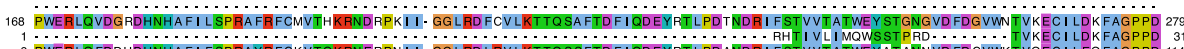

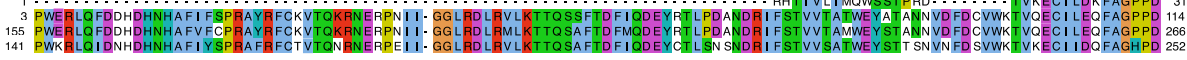

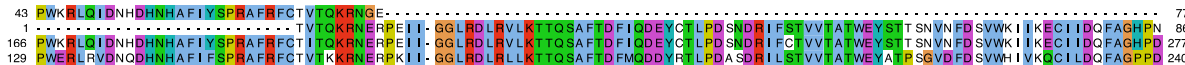

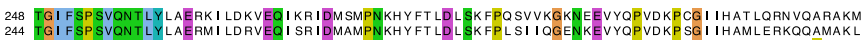

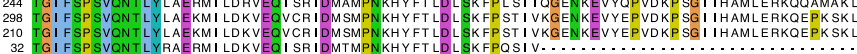

63 IGIFSPS

146 TGIFSPSVQNTLYYAERMI LDKVEQ I SRIDMTMPNKHYFT LD̈LSKFPQN IVQGENKEVYEPVDKPSG I HAMLERKQHVMAK

255 IGIFSPSVQNTLYLAERMI LDRVEQI SRI DMAMPNKHYFT LDLSKFPLSI OGENKEVYQPMDKPSG I IHAMLERKQQAMAKL

253 TGG ISPSVQNTLYLAERMI LNKVEQ I SRI DMAMPNKHYFTLDLSKKPQSI Q QGENKEVYEPVDKPSG IHAMLERKQQAMAKL

255 TGIFSP SVQNTLYLAERMI LNKVEQ I SRI DMAMPNKHYFT LDLSKKPQSI Q QGENKEVYEPVDKPSG I IHAMLERKQQAMAKL

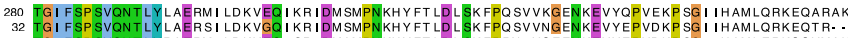

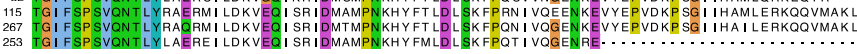

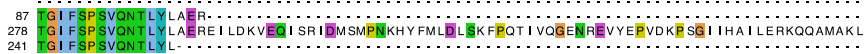

Figure S4. Protein sequence alignment of predicted uricases from 53 termite transcriptomes previously published in Buček et al. (2019). 
Table S1. Termite samples sequenced in the study.

Table S2. Relative abundance of family-level prokaryotic taxa inferred from gut metagenome and 16S rRNA amplicon data of 74 termite samples. The prokaryotic taxonomy was determined with GTDB for marker genes and with SILVA for 16S rRNA data. The relative abundance was clr-transformed to account for differences in sequencing method and sequencing depth among metagenome samples.

Table S3. Taxonomic distribution of major bacterial and archaeal groups based on relative abundance of 40 single-copy marker genes. We analyzed the marker genes present in contigs longer than $1000 \mathrm{bps}$ in $>5 \%$ of gut metagenomes. The relative abundance is represented as transcripts per million (TPM).

Table S4. Moran's I phylogenetic autocorrelation index calculated for 123 prokaryote families. Significance was assessed with 9999 random permutations. P-values $<0.05$ are indicated by asterisks.

Table S5. Relative abundance of microbial CAZymes in gut metagenomes with upward of 10000 contigs longer than 1000 bps. Relative abundance is given as transcripts per million (TPM).

Table S6. Moran's I phylogenetic autocorrelation index calculated for 211 prokaryotic CAZymes present in more than $10 \%$ of gut metagenomes. Significance was assessed with 9999 random permutations. P-values <0.05 are indicated by asterisks.

Table S7. Phylogenetic ANOVA calculated for 211 prokaryotic CAZymes present in more than $\mathbf{1 0 \%}$ of gut metagenomes. Significance was assessed with 9999 random permutations. P- 
values of phylogenetic ANOVA and pairwise comparisons were adjusted at $5 \%$ false discovery rate (FDR). The relative abundance of each CAZyme for the four termite groups are indicated by mean TPM values. Significance of pairwise comparisons between termite groups are indicated by asterisks $(* \mathrm{p}<0.05 ; * * \mathrm{p}<0.01 ; * * * \mathrm{p}<0.001)$.

Table S8. Phylogenetic ANOVA comparing the taxonomic origin of the 19 prokaryotic CAZymes found in $10 \%$ of gut metagenomes and embedded in contigs longer than 5000 bps. Significance was assessed with 9999 random permutations. The relative abundance of each CAZyme for the four termite groups are indicated by mean TPM values. Significance of pairwise comparisons between termite groups are indicated by asterisks $(* \mathrm{p}<0.05 ; * * \mathrm{p}<0.01 ; * * * \mathrm{p}<$ $0.001)$.

Table S9. Information about the 654 MAGs reconstructed in this study.

Table S10. Distribution of polysaccharide utilization loci (PULs) across the MAGs. PULs with at least one GH and Bacteroidota PULs with at least one susCD complex are shown. MAGs containing PULs with all the components are highlighted in grey.

Table S11. Moran's I phylogenetic autocorrelation index and phylogenetic ANOVA performed on the genes involved in the final steps of the lignocellulose digestion in the gut of termites. For genes composed of multiple subunits, all subunits were summed together. Significance was assessed with 9999 random permutations. P-values were adjusted at 5\% false discovery rate (FDR). The relative abundance of each gene for the four termite groups are indicated by mean TPM values. Significance of pairwise comparisons between termite groups are indicated by asterisks $(* \mathrm{p}<0.05 ; * * \mathrm{p}<0.01$; *** $\mathrm{p}<0.001)$. 
Table S12. Distribution of genes involved in reductive acetogenesis among MAGs. Distribution is shown as presence (1) and absence (0). Asterisks indicate genes that were annotated using BLASTx search against the AnnoTree database (perc. identity $>60 \%$, align. length $>100$ aa). Other genes were annotated using HMM search against the KEGG or Pfam databases. [FeFe] hydrogenase GroupA4 were annotated using the Hyddb webtool followed by manual inspection of the conserved motifs. The total number of HycB3 (PF13247) found in each MAG is shown. MAGs with almost complete reductive acetogenesis pathway ( $>5$ genes) and HDCR complex are highlighted in grey.

Table S13. Relative abundance of methyl-coenzyme $M$ reductase (mcrABG) gene complex present in metagenome contigs longer than 5000 bps. Contigs were annotated using BLASTx search against the GTDB database. Relative abundance of the gene family is shown as raw TPM.

Table S14. Distribution of genes involved in methanogenesis among MAGs. Distribution is shown as presence (1) and absence (0). Asterisks indicate genes that were annotated using BLASTx search against the AnnoTree database (perc. identity $>60 \%$, align. length $>100$ aa). Other genes were annotated using HMM search against the KEGG or Pfam databases. Highlighted MAGs have a complete Methanogenesis pathway.

Table S15. Distribution of genes involved in sulfate reducing among MAGs. Distribution is shown as presence (1) and absence (0). Asterisks indicate genes that were annotated using BLASTx search against the AnnoTree database (perc. identity $>60 \%$, align. length $>100$ aa). MAGs with complete sulfate reducing pathway are highlighted.

Table S16. Genes involved in nitrogen metabolism and fixation found in our MAGs. Distribution is shown as presence (1) and absence (0). Asterisks indicate genes that were annotated using BLASTx search against the AnnoTree database (perc. identity >60\%, align. length >100 
aa). MAGs with complete nitrogen fixation or dissimilatory nitrate reduction pathways are highlighted.

Table S17. Contigs endowed with a NifHDKENB (nifHDKENB, vnfHDKENB, or anfHDKENB) gene complex found in gut metagenomes. The relative abundance is given as raw TPM.

Table S18. Contigs endowed with a NifHDK ( $n$ ifHDK, vnfHDK, or anfHDK) gene complex found in termite gut metagenomes. The relative abundance is given as raw TPM.

Table S19. Fossil calibrations used to calibrate the time-calibrated tree of termites. 